\title{
QUANTUM FUNCTION ALGEBRAS AS QUANTUM ENVELOPING ALGEBRAS
}

\author{
FABIo Gavarini \\ Institut de Recherche Mathématique Avancée, Université "Louis Pasteur" - C.N.R.S. \\ 7, rue René Descartes, 67084 Strasbourg Cedex, France
}

\begin{abstract}
Inspired by a result in [Ga], we locate three integer forms of $F_{q}[S L(n+1)]$ over $k\left[q, q^{-1}\right]$, with a presentation by generators and relations, which for $q=1$ specialize to $U(\mathfrak{h})$, where $\mathfrak{h}$ is the Lie bialgebra of the Poisson Lie group dual to $S L(n+1)$. In sight of this we prove two PBW-like theorems for $F_{q}[S L(n+1)]$, both related to the classical PBW theorem for $U(\mathfrak{h})$.
\end{abstract}

\section{Introduction}

"Nel mezzo a una q-algebra di funzioni io ci ritrovo una tal forma intera che l'algebra di Lie dual mi doni"

\section{N. Barbecue, "Scholia"}

Let $G$ be a connected, simply connected, semisimple algebraic group over an algebraically closed field $k$ of characteristic zero, and consider on it the Sklyanin-Drinfel'd structure of Poisson group (cf. for instance $[\mathrm{DP}] \S 11$ or $[\mathrm{Ga}] \S 1$, or even [Dr]); then $\mathfrak{g}:=\operatorname{Lie}(G)$ is a Lie bialgebra, $F[G]$ is a Poisson Hopf algebra, and $U(\mathfrak{g})$ is a Poisson Hopf coalgebra. Let $H$ be the corresponding dual Poisson (algebraic) group of $G$, whose tangent Lie bialgebra $\mathfrak{h}:=\operatorname{Lie}(H)$ is the (linear) dual of $\mathfrak{g}$ : then again $F[H]$ is a Poisson Hopf algebra, and $U(\mathfrak{h})$ is a Poisson Hopf coalgebra.

The quantum group $U_{q}^{Q}(\mathfrak{g})$ of Drinfel'd and Jimbo provides a quantization of $U(\mathfrak{g})$ : namely, $U_{q}^{Q}(\mathfrak{g})$ is a Hopf algebra over $k(q)$ which has a $k\left[q, q^{-1}\right]$-form $\mathfrak{U}^{Q}(\mathfrak{g})$ which for $q \rightarrow 1$ specializes to $U(\mathfrak{g})$ as a Poisson Hopf coalgebra. Dually, by means of a Peter-Weyl type axiomatic trick one constructs a Hopf algebra $F_{q}^{P}[G]$ of matrix coefficients of $U_{q}^{Q}(\mathfrak{g})$ with a $k\left[q, q^{-1}\right]$-form $\mathfrak{F}^{P}[G]$ which specializes to $F[G]$, as a Poisson Hopf algebra, for

1991 Mathematics Subject Classification: Primary 17B37, 81R50

Partially supported by a post-doc fellowship of the Consiglio Nazionale delle Ricerche (Italy) 
$q \rightarrow 1$. So far the quantization only dealt with the Poisson group $G$; the dual group $H$ is involved defining a different $k\left[q, q^{-1}\right]$-form $\mathcal{U}^{P}\left(\mathfrak{g}\right.$ ) (of a quantum group $U_{q}^{P}(\mathfrak{g})$ ) which specializes to $F[H]$ (as a Poisson Hopf algebra) for $q \rightarrow 1$ (cf. [DP] or [DKP]). In a dual fashion, it is proved in [Ga] — in a wider context - that the dual (in the Hopf sense) quantum function algebra $F_{q}^{Q}[G]$ has a $k\left[q, q^{-1}\right]$-integer form $\mathcal{F}^{Q}[G]$ which for $q \rightarrow 1$ specializes to $U(\mathfrak{h})$, as a Poisson Hopf coalgebra. Therefore quantum function algebras can also be thought of as quantum enveloping algebras, whence the title of the paper.

In this paper we stick to the case of the group $G=S L(n+1)$.

Our first goal is to relate the latter result above with the well-known presentation of $F_{q}^{P}[S L(n+1)]$ by generators and relations (cf. [FRT]): namely, inspired by the definition of $\mathcal{F}^{Q}[G]$ and $\mathcal{F}^{P}[G]$, we define two $k\left[q, q^{-1}\right]$-integer forms $\widetilde{F}_{q}^{Q}[S L(n+1)]$ and $\widetilde{F}_{q}^{P}[S L(n+1)]$ (along with a third one, $\widetilde{F}_{q}[S L(n+1)]$ ) of $F_{q}^{P}[S L(n+1)]$; these inherit a presentation by generators and relations, which enables us to prove that they specialize to $U(\mathfrak{h}$ ) (as a Poisson Hopf coalgebra) for $q \rightarrow 1$. As a second step, since for $U(\mathfrak{h})$ one has the PoincaréBirkhof-Witt (PBW in short) theorem which provides "monomial" basis, because of the previous result we are led to look for PBW-like theorems for $F_{q}^{P}[S L(n+1)]$ : we provide two of them, both closely related with the classical PBW theorem for $U(\mathfrak{h})$.

The paper is organized as follows. Sections 1,2 are introductory. Sections 3, 4 are devoted to integer forms of $F_{q}^{P}[S L(n+1)]$ and their specialization. Section 5 is an excursus, where we explain the relation among the constructions and results in this paper and those in [Ga]: this is one of the main motivation of this work; on the other hand, this section can be skipped without affecting the comprehension of the rest of the paper, which is completely self-contained. In section 6 we briefly outline the extension of the previous results to the quantum function algebra $F_{q}[G L(n+1)]$. Finally, section 7 deals with PBW theorems.

\section{$\S 1$ The universal enveloping algebra $U(\mathfrak{h})$}

A presentation of $U(\mathfrak{h})$ by generators and relations (in the general case) can be found in [Ga], $\S 1$. When $G=S L(n+1)$, it reads as follows.

$U(\mathfrak{h})$ is the associative $k$-algebra with 1 generated by $\mathrm{f}_{1}, \ldots, \mathrm{f}_{n}, \mathrm{~h}_{1}, \ldots, \mathrm{h}_{n}, \mathrm{e}_{1}, \ldots, \mathrm{e}_{n}$ (which are to be thought of as "Chevalley generators") with relations

$$
\begin{array}{cr}
\mathrm{h}_{i} \mathrm{~h}_{j}-\mathrm{h}_{j} \mathrm{~h}_{i}=0 & \forall i, j \\
\mathrm{~h}_{i} \mathrm{f}_{j}-\mathrm{f}_{j} \mathrm{~h}_{i}=\left(2 \delta_{i, j}-\delta_{i-1, j}-\delta_{i+1, j}\right) \mathrm{f}_{j} & \forall i, j \\
\mathrm{~h}_{i} \mathrm{e}_{j}-\mathrm{e}_{j} \mathrm{~h}_{i}=\left(2 \delta_{i, j}-\delta_{i-1, j}-\delta_{i+1, j}\right) \mathrm{e}_{j} & \forall i, j \\
\mathrm{f}_{i} \mathrm{f}_{j}-\mathrm{f}_{j} \mathrm{f}_{i}=0 & \forall i, j:|i-j|>1 \\
\mathrm{e}_{i} \mathrm{e}_{j}-\mathrm{e}_{j} \mathrm{e}_{i}=0 & \forall i, j:|i-j|>1 \\
\mathrm{f}_{i}^{2} \mathrm{f}_{j}-2 \mathrm{f}_{i} \mathrm{f}_{j} \mathrm{f}_{i}+\mathrm{f}_{j} \mathrm{f}_{i}^{2}=0 & \forall|i-j|=1 \\
\mathrm{e}_{i}^{2} \mathrm{e}_{j}-2 \mathrm{e}_{i} \mathrm{e}_{j} \mathrm{e}_{i}+\mathrm{e}_{j} \mathrm{e}_{i}^{2}=0 & \forall|i-j|=1 \\
\mathrm{f}_{i} \mathrm{e}_{j}-\mathrm{e}_{j} \mathrm{f}_{i}=0 & \forall i, j
\end{array}
$$


Furthermore $U(\mathfrak{h})$ has a Poisson Hopf coalgebra structure given by

$$
\begin{array}{rlrl}
\Delta\left(\mathrm{f}_{i}\right)=\mathrm{f}_{i} \otimes 1+1 \otimes \mathrm{f}_{i}, & S\left(\mathrm{f}_{i}\right)=-\mathrm{f}_{i}, & \epsilon\left(\mathrm{f}_{i}\right)=0 \\
\Delta\left(\mathrm{h}_{i}\right)=\mathrm{h}_{i} \otimes 1+1 \otimes \mathrm{h}_{i}, & S\left(\mathrm{~h}_{i}\right)=-\mathrm{h}_{i}, & & \epsilon\left(\mathrm{h}_{i}\right)=0 \\
\Delta\left(\mathrm{e}_{i}\right)=\mathrm{e}_{i} \otimes 1+1 \otimes \mathrm{e}_{i}, & S\left(\mathrm{e}_{i}\right)=-\mathrm{e}_{i}, & & \epsilon\left(\mathrm{e}_{i}\right)=0
\end{array}
$$

for all $i=1, \ldots, n$, and by

$$
\begin{gathered}
\delta\left(\mathrm{f}_{i}\right)=\mathrm{h}_{i} \wedge \mathrm{f}_{i}+2 \cdot\left(\sum_{j=1}^{i-1} \mathrm{f}_{i+1, j} \wedge \mathrm{e}_{j, i}+\sum_{j=i+2}^{n+1} \mathrm{e}_{i+1, j} \wedge \mathrm{f}_{j, i}\right) \\
\delta\left(\mathrm{h}_{i}\right)=4 \cdot\left(\sum_{j=1}^{i-1} \mathrm{f}_{i, j} \wedge \mathrm{e}_{j, i}+\sum_{j=i+1}^{n+1} \mathrm{e}_{i, j} \wedge \mathrm{f}_{j, i}-\sum_{j=1}^{i} \mathrm{f}_{i+1, j} \wedge \mathrm{e}_{j, i+1}-\sum_{j=i+2}^{n+1} \mathrm{e}_{i+1, j} \wedge \mathrm{f}_{j, i+1}\right) \\
\delta\left(\mathrm{e}_{i}\right)=\mathrm{e}_{i} \wedge \mathrm{h}_{i}+2 \cdot\left(\sum_{j=1}^{i-1} \mathrm{e}_{j, i+1} \wedge \mathrm{f}_{i, j}+\sum_{j=i+2}^{n+1} \mathrm{f}_{j, i+1} \wedge \mathrm{e}_{i, j}\right)
\end{gathered}
$$

for all $i=1, \ldots, n$, where $x \wedge y:=x \otimes y-y \otimes x$ and the symbols $\mathrm{f}_{h, k}, \mathrm{e}_{h, k}$, have the following meaning:

$$
\begin{array}{lll}
\mathrm{e}_{i, i+1}:=\mathrm{e}_{i}, & \mathrm{e}_{i, j}:=-\left[\mathrm{e}_{i, j-1}, \mathrm{e}_{j-1, j}\right]=\left[\mathrm{e}_{j-1, j}, \mathrm{e}_{i, j-1}\right] & \forall i<j-1 \\
\mathrm{f}_{j+1, j}:=\mathrm{f}_{j}, & \mathrm{f}_{j, i}:=\left[\mathrm{f}_{j-1, i}, \mathrm{f}_{j, j-1}\right]=-\left[\mathrm{f}_{j, j-1}, \mathrm{f}_{j-1, i}\right] & \forall j>i+1
\end{array}
$$

the symbol [, ] denoting the usual commutator. In fact, if $M_{i, j}(i, j \in\{1,2, \ldots, n+1\})$ denotes the square matrix of size $n+1$ with a 1 as $(i, j)$-th entry and all other entries equal to 0 , the recipe $\mathrm{e}_{h} \mapsto M_{h, h+1} \forall h=1, \ldots, n$ (resp. $\mathrm{f}_{h} \mapsto M_{h+1, h} \forall h=1, \ldots, n$ ) gives an isomorphism among the Lie subalgebra of $\mathfrak{h}$ generated by the $e_{h}$ 's (resp. $f_{h}$ 's) and the Lie algebra $\mathfrak{n}_{+}$(resp. $\mathfrak{n}_{+}$) of upper (resp. lower) triangular square matrix of size $n+1$; then for $h<k$ the element $\mathrm{e}_{h, k}$ corresponds to the matrix $(-1)^{k-h-1} M_{h, k}$, and it is the root vector $\mathrm{e}_{\gamma}$ - in the notation of $[\mathrm{Ga}]$ - associated to the positive root $\gamma=\sum_{i=h}^{k-1} \alpha_{i}$ (the $\alpha_{i}$ 's being the simple roots of $S L(n+1)$ ), and for $h>k$ the element $\mathrm{f}_{h, k}$ corresponds to the matrix $(-1)^{k-h-1} M_{h, k}$, and it is the root vector $\mathrm{f}_{\gamma}$ - in the notation of [Ga] associated to the negative root $-\gamma=-\sum_{i=k}^{h-1} \alpha_{i}$, hence corresponding to $M_{h, k}$. Actually, one can also make different choices for such root vectors, but for the condition that when one of them - say $\mathrm{e}_{\gamma}$ — is multiplied by a scalar $c \in k \backslash\{0\}$ then the opposite one $\mathrm{f}_{\gamma}$ in our case - is multiplied by the inverse scalar $c^{-1}$; hence the right-hand-side part in the above formulae expressing $\delta$ does not change.

\section{$\S 2$ The quantum function algebra $F_{q}^{P}[S L(n+1)]$}

Let $U_{q}^{Q}(\mathfrak{s l}(n+1))$ be the quantized universal enveloping algebra of Drifel'd and Jimbo (cf. [Ji] or [DL], or $\S 5.2$ later on). Let $F_{q}^{P}[S L(n+1)]$ be its restricted dual Hopf algebra: it is known (cf. [APW], Appendix) that $F_{q}^{P}[S L(n+1)]$ has the following presentation: it is 
the unital associative $k(q)$-algebra generated by $\left\{\rho_{i j} \mid i, j=1, \ldots, n+1\right\}$ with relations

$$
\begin{array}{ccrl}
\rho_{i j} \rho_{i k}=q \rho_{i k} \rho_{i j}, \quad \rho_{i k} \rho_{h k} & =q \rho_{h k} \rho_{i k} & & \forall j<k, i<h \\
\rho_{i l} \rho_{j k}=\rho_{j k} \rho_{i l}, \quad \rho_{i k} \rho_{j l}-\rho_{j l} \rho_{i k} & =\left(q-q^{-1}\right) \rho_{i l} \rho_{j k} & & \forall i<j, k<l \\
\operatorname{det}_{q}\left(\rho_{i j}\right)=1 & &
\end{array}
$$

where $\operatorname{det}_{q}$ denotes the so-called quantum determinant, defined as

$$
\operatorname{det}_{q}\left(\rho_{i j}\right):=\sum_{\sigma \in S_{n+1}}(-q)^{l(\sigma)} \rho_{1, \sigma(1)} \rho_{2, \sigma(2)} \cdots \rho_{n+1, \sigma(n+1)} .
$$

The comultiplication $\Delta$, the counit $\epsilon$, and the antipode $S$ are given by

$$
\begin{array}{cc}
\Delta\left(\rho_{i j}\right)=\sum_{k=1}^{n} \rho_{i k} \otimes \rho_{k j} & \forall i, j=1, \ldots, n+1 \\
\epsilon\left(\rho_{i j}\right)=\delta_{i j} & \forall i, j=1, \ldots, n+1 \\
S\left(\rho_{i j}\right)=(-q)^{j-i} \operatorname{det}_{q}\left(\left(\rho_{h k}\right)_{h \neq j}^{k \neq i} h\right) & \forall i, j=1, \ldots, n+1 .
\end{array}
$$

$\S 3$ The integer forms $\widetilde{F}_{q}^{Q}[S L(n+1)]$ and $\widetilde{F}_{q}^{P}[S L(n+1)]$

Definition 3.1. We define $\widetilde{F}_{q}^{Q}[S L(n+1)]$ to be the $k\left[q, q^{-1}\right]$-subalgebra (with 1 ) of $F_{q}^{P}[S L(n+1)]$ generated by the elements

$$
\varphi_{i}:=\frac{\rho_{i i}-\rho_{i+1, i+1}}{q-1}, \quad r_{i j}:=\left(q-q^{-1}\right)^{\delta_{i j}-1} \rho_{i j} \quad \forall i, j=1, \ldots, n+1 .
$$

3.2 Presentation of $\widetilde{F}_{q}^{Q}[S L(n+1)]$. The presentation of $F_{q}^{P}[S L(n+1)]$ above induces a similar presentation of $\widetilde{F}_{q}^{Q}[S L(n+1)]$ : it is the associative $k\left[q, q^{-1}\right]$-algebra with 1 given by generators $\varphi_{i}, r_{i j}$, and relations

$$
\begin{aligned}
& r_{i j} r_{i k}=q r_{i k} r_{i j}, \quad r_{i k} r_{h k}=q r_{h k} r_{i k} \\
& r_{i l} r_{j k}=r_{j k} r_{i l}, \quad r_{i k} r_{j l}-r_{j l} r_{i k}=\left(q-q^{-1}\right)^{1+\delta_{i k}+\delta_{j l}-\delta_{i l}-\delta_{j k}} r_{i l} r_{j k} \quad \forall i<j, k<l \\
& \widetilde{\operatorname{det}_{q}}\left(r_{i j}\right)=1
\end{aligned}
$$

(where $\widetilde{d e t}_{q}$ is defined as

$$
\widetilde{\operatorname{det}}_{q}\left(\left(x_{r s}\right)_{r, s=1, \ldots, N}\right):=\sum_{\sigma \in S_{N}}(-q)^{l(\sigma)}\left(q-q^{-1}\right)^{e(\sigma)} x_{1, \sigma(1)} x_{2, \sigma(2)} \cdots x_{N, \sigma(N)}
$$

where $e(\sigma):=\sum_{t=1}^{N}\left(1-\delta_{t, \sigma(t)}\right)$

$$
\begin{array}{crr}
(q-1) \varphi_{i}=r_{i i}-r_{i+1, i+1} & \forall i=1, \ldots, n \\
\varphi_{i} r_{j k}-r_{j k} \varphi_{i}=0 & \forall j<i, k>i+1, \forall j>i+1, k<i \\
\varphi_{i} r_{j k}-r_{j k} \varphi_{i}=(q-1)^{1+\delta_{j k}}\left(1+q^{-1}\right)^{2+\delta_{j k}}\left(r_{i+1, k} r_{j, i+1}-r_{i k} r_{j i}\right) & \forall j<i, k<i
\end{array}
$$




$$
\begin{array}{cr}
\varphi_{i} r_{j k}-r_{j k} \varphi_{i}=-(q-1)^{1+\delta_{j k}}\left(1+q^{-1}\right)^{2+\delta_{j k}}\left(r_{i+1, k} r_{j, i+1}-r_{i k} r_{j i}\right) & \forall j>i+1, k>i+1 \\
\varphi_{i} r_{j i}-r_{j i} \varphi_{i}=-r_{i i} r_{j i}+(q-1)\left(1+q^{-1}\right)^{2} r_{j, i+1} r_{i+1, i} & \forall j<i \\
\varphi_{i} r_{j i}-r_{j i} \varphi_{i}=r_{j i} r_{i i} & \forall j>i+1 \\
\varphi_{i} r_{j, i+1}-r_{j, i+1} \varphi_{i}=r_{i+1, i+1} r_{j, i+1} & \forall j<i \\
\varphi_{i} r_{j, i+1}-r_{j, i+1} \varphi_{i}=-r_{j, i+1} r_{i+1, i+1}+(q-1)\left(1+q^{-1}\right)^{2} r_{i, i+1} r_{j i} & \forall j<i \\
\varphi_{i} r_{i j}-r_{i j} \varphi_{i}=-r_{i i} r_{i j}+(q-1)\left(1+q^{-1}\right)^{2} r_{i, i+1} r_{i+1, j} & \forall j<i \\
\varphi_{i} r_{i j}-r_{i j} \varphi_{i}=r_{i j} r_{i i} & \forall j>i+1 \\
\varphi_{i} r_{i+1, j}-r_{i+1, j} \varphi_{i}=r_{i+1, i+1} r_{i+1, j} & \forall j<i \\
\varphi_{i} r_{i+1, j}-r_{i+1, j} \varphi_{i}=-r_{i+1, j} r_{i+1, i+1}+(q-1)\left(1+q^{-1}\right)^{2} r_{i, j} r_{i+1, i} & \forall j>i+1 \\
\varphi_{i} r_{i i}-r_{i i} \varphi_{i}=(q-1)^{2}\left(1+q^{-1}\right)^{3} r_{i+1, i} r_{i, i+1} & \forall i \\
\varphi_{i} r_{i+1, i+1}-r_{i+1, i+1} \varphi_{i}=(q-1)^{2}\left(1+q^{-1}\right)^{3} r_{i+1, i} r_{i, i+1} & \forall i \\
\varphi_{i} r_{i, i+1}-r_{i, i+1} \varphi_{i}=r_{i, i+1} r_{i i}+r_{i+1, i+1} r_{i, i+1} & \forall i \\
\varphi_{i} r_{i+1, i}-r_{i+1, i} \varphi_{i}=r_{i+1, i} r_{i i}+r_{i+1, i+1} r_{i+1, i} & \forall i
\end{array}
$$

$\varphi_{i} \varphi_{j}-\varphi_{j} \varphi_{i}=$

$$
=(q-1)\left(1+q^{-1}\right)^{3}\left(r_{i j} r_{j i}+r_{i+1, j+1} r_{j+1, i+1}-r_{i, j+1} r_{j+1, i}-\left(1-\delta_{i+1, j}\right) r_{i+1, j} r_{j, i+1}\right) \forall i, j
$$

Moreover, from the very definitions we also get that $\widetilde{F}_{q}^{Q}[S L(n+1)]$ is a Hopf subalgebra of $F_{q}^{P}[S L(n+1)]$, with Hopf structure uniquely determined by the following formulae:

$$
\left.\begin{array}{cc}
\Delta\left(r_{i j}\right)=r_{i i} \otimes r_{i j}+r_{i j} \otimes r_{j j}+(q-1)\left(1+q^{-1}\right) \sum_{\substack{k=1 \\
k \neq i, j}}^{n+1} r_{i k} \otimes r_{k j} & \forall i \neq j \\
\Delta\left(r_{i i}\right)=r_{i i} \otimes r_{i i}+(q-1)^{2}\left(1+q^{-1}\right)^{2} \sum_{\substack{k=1 \\
k \neq i}}^{n+1} r_{i k} \otimes r_{k j} & \forall i \\
\Delta\left(\varphi_{i}\right)=r_{i, i} \otimes \varphi_{i}+\varphi_{i} \otimes r_{i+1, i+1}+(q-1)\left(1+q^{-1}\right)^{2}\left(\sum_{\substack{k=1 \\
k \neq i}}^{n+1} r_{i, k} \otimes r_{k, i}-\sum_{\substack{k=1 \\
k \neq i+1}}^{n+1} r_{i+1, k} \otimes r_{k, i+1}\right.
\end{array}\right) \forall i
$$


Remark 3.3. It is clear by definition that $\widetilde{F}_{q}^{Q}[S L(n+1)]$ is a $k\left[q, q^{-1}\right]$-integer form of $F_{q}^{P}[S L(n+1)]$ : in other words, it is a Hopf $k\left[q, q^{-1}\right]$-subalgebra of $F_{q}^{P}[S L(n+1)]$ which is flat as a $k\left[q, q^{-1}\right]$-module and is such that $k(q) \otimes_{k\left[q, q^{-1}\right]} \widetilde{F}_{q}^{Q}[S L(n+1)] \cong F_{q}^{P}[S L(n+1)]$ as Hopf $k(q)$-algebras.

Definition 3.4. We define $\widetilde{F}_{q}^{P}[S L(n+1)]$ to be the $k\left[q, q^{-1}\right]$-subalgebra (with 1 ) of $F_{q}^{P}[S L(n+1)]$ generated by the elements

$$
\psi_{i}:=\frac{\rho_{11} \rho_{22} \cdots \rho_{i i}-1}{q-1}, \quad r_{i j}:=\left(q-q^{-1}\right)^{\delta_{i j}-1} \rho_{i j} \quad \forall i, j=1, \ldots, n+1 .
$$

3.5 Presentation of $\widetilde{F}_{q}^{P}[S L(n+1)]$. The presentation of $F_{q}^{P}[S L(n+1)]$ above induces a similar presentation of $\widetilde{F}_{q}^{P}[S L(n+1)]$ : in fact the latter is the associative $k\left[q, q^{-1}\right]$-algebra with 1 given by generators $\psi_{i}, r_{i j}$, and relations

$$
\begin{aligned}
& r_{i j} r_{i k}=q r_{i k} r_{i j}, \quad r_{i k} r_{h k}=q r_{h k} r_{i k} \quad \forall j<k, i<h \\
& r_{i l} r_{j k}=r_{j k} r_{i l}, \quad r_{i k} r_{j l}-r_{j l} r_{i k}=\left(q-q^{-1}\right)^{1+\delta_{i k}+\delta_{j l}-\delta_{i l}-\delta_{j k}} r_{i l} r_{j k} \quad \forall i<j, k<l \\
& \widetilde{\operatorname{det}_{q}}\left(r_{i j}\right)=1 \\
& (q-1) \psi_{i}=r_{11} r_{22} \cdots r_{i i}-1 \quad \forall i=1, \ldots, n+1 \\
& \psi_{i} r_{j k}=q^{1-\eta(i, j, k)-\zeta(i, j, k)} r_{j k} \psi_{i}+\theta(i, j, k) \cdot r_{j k}+(q-1)^{1+\delta_{j k}}\left(1+q^{-1}\right)^{2+\delta_{j k}} \text {. } \\
& \cdot \sum_{s=1}^{i}(\eta(i, j, k)-\zeta(i, j, k)) r_{11} \cdots r_{s-1, s-1} r_{s k} r_{j s} r_{s+1, s+1} \cdots r_{i i} \quad \forall i, j, k
\end{aligned}
$$

(where $\theta(i, j, k):=1 \forall j \leq i<k$ or $k \leq i<j, \eta(i, j, k):=1 \forall i<j \wedge k, \zeta(i, j, k):=1$ $\forall i \geq j \vee k$, whilst $\theta(i, j, k), \eta(i, j, k)$ and $\zeta(i, j, k)$ are zero in the other cases)

$$
\begin{gathered}
\psi_{n+1}=-\sum_{\substack{\sigma \in S_{n+1} \\
\sigma \neq i d}}(-q)^{l(\sigma)}(q-1)^{e(\sigma)-1}\left(1+q^{-1}\right)^{e(\sigma)} r_{1, \sigma(1)} r_{2, \sigma(2)} \cdots r_{n+1, \sigma(n+1)} \\
\psi_{i} \psi_{j}-\psi_{j} \psi_{i}=(q-1)\left(1+q^{-1}\right)^{3} . \\
\cdot \sum_{k=i+1}^{j} \sum_{s=1}^{i} r_{11} r_{22} \cdots r_{k-1, k-1} \cdot r_{11} r_{22} \cdots r_{s-1, s-1} r_{s k} r_{k s} r_{s+1, s+1} \cdots r_{i i} \cdot r_{k+1, k+1} r_{k+2, k+2} \cdots r_{j j} \forall i<j
\end{gathered}
$$

Furthermore, from the very definitions we also get that $\widetilde{F}_{q}^{P}[S L(n+1)]$ is a Hopf subalgebra of $F_{q}^{P}[S L(n+1)]$, with Hopf structure uniquely determined by the following formulae:

$$
\begin{array}{cc}
\Delta\left(r_{i j}\right)=r_{i i} \otimes r_{i j}+r_{i j} \otimes r_{j j}+(q-1)\left(1+q^{-1}\right) \sum_{\substack{k=1 \\
k \neq i, j}}^{n+1} r_{i k} \otimes r_{k j} & \forall i \neq j \\
\Delta\left(r_{i i}\right)=r_{i i} \otimes r_{i i}+(q-1)^{2}\left(1+q^{-1}\right)^{2} \sum_{\substack{k=1 \\
k \neq i}}^{n+1} r_{i k} \otimes r_{k j} & \forall i
\end{array}
$$




$$
\Delta\left(\psi_{i}\right)=\left(1+q^{-1}\right) \cdot \sum_{s}\left(q-q^{-1}\right)^{2 \cdot N(s)-1} \prod_{k=1}^{i} r_{k, s(k)} \otimes r_{s(k), k}+\psi_{i} \otimes r_{1,1} r_{2,2} \cdots r_{i, i}+1 \otimes \psi_{i} \forall i
$$

(where $s$ ranges over all maps $s:\{1,2, \ldots, i\} \rightarrow\{1,2, \ldots, n+1\}$ such that $s(j) \neq j$ for some $j \in\{1,2, \ldots, i\}$, and $\left.N(s):=\sum_{j=1}^{i}\left(1-\delta_{j, s(j)}\right)\right)$

$$
\begin{gathered}
S\left(r_{i j}\right)=(-q)^{j-i} \widetilde{\operatorname{det}}_{q}\left(\left(r_{h k}\right)_{\substack{k \neq i \\
h \neq j}}\right) \\
S\left(\psi_{i}\right)=-\psi_{i}+\mathcal{O}(q-1) \\
\varepsilon\left(r_{i j}\right)=\delta_{i j}, \quad \varepsilon\left(\psi_{i}\right)=0
\end{gathered}
$$

where $\mathcal{O}(q-1)$ denotes some element of $(q-1) \cdot \widetilde{F}_{q}^{P}[S L(n+1)]$. To give an example, we show that $S\left(\psi_{i}\right)=-\psi_{i}+\mathcal{O}(q-1)$. By definition we have

$$
S\left(\psi_{i}\right)=S\left(\frac{r_{11} r_{22} \cdots r_{i i}-1}{q-1}\right)=\frac{S\left(r_{i i}\right) \cdots S\left(r_{22}\right) S\left(r_{11}\right)-1}{q-1} ;
$$

but

$$
\begin{aligned}
S\left(r_{j j}\right) & =\widetilde{\operatorname{det}}_{q}\left(\left(r_{h k}\right)_{h, k \neq j}\right)= \\
& =r_{11} r_{22} \cdots r_{j-1, j-1} \cdot r_{j+1, j+1} \cdots r_{n+1, n+1}+\mathcal{O}\left((q-1)^{2}\right)=\prod_{\substack{s=1 \\
s \neq j}}^{n+1} r_{s s}+\mathcal{O}\left((q-1)^{2}\right)
\end{aligned}
$$

for all $j$, and

$$
1=\widetilde{\operatorname{det}}_{q}\left(r_{h k}\right)=r_{11} r_{22} \cdots r_{n+1, n+1}+\mathcal{O}\left((q-1)^{2}\right)
$$

therefore

$$
S\left(\psi_{i}\right)=\frac{\prod_{\substack{s=1 \\ s \neq i}}^{n+1} r_{s s} \cdot \prod_{\substack{s=1 \\ s \neq i-1}}^{n+1} r_{s s} \cdots \prod_{\substack{s=1 \\ s \neq 2}}^{n+1} r_{s s} \cdot \prod_{\substack{s=1 \\ s \neq 1}}^{n+1} r_{s s}-\left(\prod_{s=1}^{n+1} r_{s s}\right)^{i}}{q-1}+\mathcal{O}(q-1)
$$

now using the fact that $r_{h h} r_{k k}=r_{k k} r_{h h}+\mathcal{O}\left((q-1)^{3}\right)$ we get

$$
S\left(\psi_{i}\right)=\prod_{\substack{s=1 \\ s \neq i}}^{n+1} r_{s s} \cdot \prod_{\substack{s=1 \\ s \neq i-1}}^{n+1} r_{s s} \cdots \prod_{\substack{s=1 \\ s \neq 2}}^{n+1} r_{s s} \cdot \prod_{\substack{s=1 \\ s \neq 1}}^{n+1} r_{s s} \cdot \frac{1-r_{11} r_{22} \cdots r_{i i}}{q-1}+\mathcal{O}(q-1)
$$

and finally, since $r_{j j}=1+\mathcal{O}(q-1)$ (as one easily gets from relations $(q-1) \psi_{s}=$ $r_{11} r_{22} \cdots r_{s s}-1$ ), we find

$$
S\left(\psi_{i}\right)=\frac{1-r_{11} r_{22} \cdots r_{i i}}{q-1}+\mathcal{O}(q-1)=-\psi_{i}+\mathcal{O}(q-1), \quad \text { q.e.d. }
$$


Remark 3.6. Here again, it is clear — by definition and by the description of the Hopf structure - that $\widetilde{F}_{q}^{P}[S L(n+1)]$ is a $k\left[q, q^{-1}\right]$-integer form of $F_{q}^{P}[S L(n+1)]$.

Definition 3.7. We define $\widetilde{F}_{q}[S L(n+1)]$ to be the $k\left[q, q^{-1}\right]$-subalgebra (with 1 ) of $F_{q}^{P}[S L(n+1)]$ generated by the elements

$$
\chi_{i}:=\frac{\rho_{i i}-1}{q-1}, \quad r_{i j}:=\left(q-q^{-1}\right)^{\delta_{i j}-1} \rho_{i j} \quad \forall i, j=1, \ldots, n+1 .
$$

3.8 Presentation of $\widetilde{F}_{q}[S L(n+1)]$. Again, we have a presentation of $\widetilde{F}_{q}[S L(n+1)]$ : it is the associative $k\left[q, q^{-1}\right]$-algebra with 1 given by generators $\chi_{i}, r_{i j}$, and relations

$$
\begin{array}{cr}
r_{i j} r_{i k}=q r_{i k} r_{i j}, \quad r_{i k} r_{h k}=q r_{h k} r_{i k} & \forall j<k, i<h \\
r_{i l} r_{j k}=r_{j k} r_{i l}, \quad r_{i k} r_{j l}-r_{j l} r_{i k}=\left(q-q^{-1}\right)^{1+\delta_{i k}+\delta_{j l}-\delta_{i l}-\delta_{j k}} r_{i l} r_{j k} & \forall i<j, k<l \\
\widetilde{d e t} t_{q}\left(r_{i j}\right)=1 & \forall i=1, \ldots, n+1 \\
(q-1) \chi_{i}=r_{i i}-1 & \forall j<i<k, \forall j>i>k \\
\chi_{i} r_{j k}-r_{j k} \chi_{i}=0 & \forall j<i \\
\chi_{i} r_{j i}-r_{j i} \chi_{i}=-r_{i i} r_{j i} & \forall j>i \\
\chi_{i} r_{j i}-r_{j i} \chi_{i}=+r_{i i} r_{j i} & \forall i \\
\chi_{i} r_{i i}-r_{i i} \chi_{i}=0 & \forall k<i \\
\chi_{i} r_{i k}-r_{i k} \chi_{i}=-r_{i i} r_{i k} & \forall k>i \\
\chi_{i} r_{i k}-r_{i k} \chi_{i}=+r_{i i} r_{i k} & \forall j, k<i \\
\chi_{i} r_{j k}-r_{j k} \chi_{i}=-(q-1)^{2}\left(1+q^{-1}\right)^{3} r_{i i} r_{i k} & \forall j, k>i \\
\chi_{i} r_{j k}-r_{j k} \chi_{i}=+(q-1)^{2}\left(1+q^{-1}\right)^{3} r_{i i} r_{i k} & \forall i \leq j \\
\chi_{i} \chi_{j}-\chi_{j} \chi_{i}=\left(1-\delta_{i j}\right) \cdot(q-1)\left(1+q^{-1}\right)^{3} r_{i j} r_{j i} & \\
\sum_{i=1}^{n+1} r_{1,1} r_{2,2} \cdots r_{i-1, i-1} \chi_{i}= & \\
\sum_{\sigma \in S_{n+1} \backslash\{1\}} &
\end{array}
$$

A straightforward verification shows that $\widetilde{F}_{q}[S L(n+1)]$ is also a Hopf subalgebra of $F_{q}^{P}[S L(n+1)]$, whose Hopf structure is given by formulae

$$
\begin{array}{cc}
\Delta\left(r_{i j}\right)=r_{i i} \otimes r_{i j}+r_{i j} \otimes r_{j j}+(q-1)\left(1+q^{-1}\right) \sum_{\substack{k=1 \\
k \neq i, j}}^{n+1} r_{i k} \otimes r_{k j} & \forall i \neq j \\
\Delta\left(r_{i i}\right)=r_{i i} \otimes r_{i i}+(q-1)^{2}\left(1+q^{-1}\right)^{2} \sum_{\substack{k=1 \\
k \neq i}}^{n+1} r_{i k} \otimes r_{k j} & \forall i
\end{array}
$$




$$
\begin{aligned}
& \Delta\left(\chi_{i}\right)=r_{i i} \otimes \chi_{i}+(q-1)\left(1+q^{-1}\right)^{2} \sum_{\substack{k=1 \\
k \neq i}}^{n+1} r_{i, k} \otimes r_{k, i} \\
& S\left(r_{i j}\right)=(-q)^{j-i} \widetilde{\operatorname{det}}_{q}\left(\left(r_{h k}\right)_{h \neq j}^{k \neq i}\right) \\
& S\left(\chi_{i}\right)=-r_{1,1} r_{2,2} \cdots r_{i-1, i-1} \chi_{i} r_{i+2, i+2} \cdots r_{n+1, n+1}+ \\
& +\sum_{\sigma \in S_{n} \backslash\{1\}}(-q)^{l(\sigma)}(q-1)^{e(\sigma)-1}\left(1+q^{-1}\right)^{e(\sigma)} \prod_{\substack{j=1 \\
j \neq i \\
\sigma(j) \neq i}}^{n+1} r_{j, \sigma(j)}- \\
& -\sum_{\sigma \in S_{n+1} \backslash\{1\}}(-q)^{l(\sigma)}(q-1)^{e(\sigma)-1}\left(1+q^{-1}\right)^{e(\sigma)} \prod_{j=1}^{n+1} r_{j, \sigma(j)} \quad \forall i \\
& \varepsilon\left(r_{i j}\right)=\delta_{i j}, \quad \varepsilon\left(\chi_{i}\right)=0
\end{aligned}
$$

Remark 3.9. By definition and by the description of its Hopf structure we see at once that $\widetilde{F}_{q}[S L(n+1)]$ is a $k\left[q, q^{-1}\right]$-integer form of $F_{q}^{P}[S L(n+1)]$.

\section{$\S 4$ The main theorem: specialization results}

Since the integer forms we are dealing with are Hopf algebras over the ring $k\left[q, q^{-1}\right]$, we can consider their specialization at $q=1$, namely the Hopf $k$-algebras

$$
\begin{gathered}
\widetilde{F}_{1}^{Q}[S L(n+1)]:=\widetilde{F}_{q}^{Q}[S L(n+1)] /(q-1) \widetilde{F}_{q}^{Q}[S L(n+1)], \\
\widetilde{F}_{1}^{P}[S L(n+1)]:=\widetilde{F}_{q}^{P}[S L(n+1)] /(q-1) \widetilde{F}_{q}^{P}[S L(n+1)], \\
\widetilde{F}_{1}[S L(n+1)]:=\widetilde{F}_{q}[S L(n+1)] /(q-1) \widetilde{F}_{q}[S L(n+1)] .
\end{gathered}
$$

Our main result is the following:

Theorem 4.1. The Hopf $k$-algebras $\widetilde{F}_{1}^{Q}[S L(n+1)], \widetilde{F}_{1}^{P}[S L(n+1)]$, and $\widetilde{F}_{1}[S L(n+1)]$, are Poisson Hopf coalgebras isomorphic to $U(\mathfrak{h})$. In other words, $\widetilde{F}_{q}^{Q}[S L(n+1)], \widetilde{F}_{q}^{P}[S L(n+1)]$, and $\widetilde{F}_{q}[S L(n+1)]$ all specialize to the Poisson Hopf coalgebra $U(\mathfrak{h})$.

Proof. Consider $\widetilde{F}_{1}^{Q}[S L(n+1)]$; it inherits from $\widetilde{F}_{q}^{Q}[S L(n+1)]$ the following presentation (which is obtained from that of $\widetilde{F}_{q}^{Q}[S L(n+1)]$ by setting $q=1$ ): it is the unital associative $k$-algebra with generators $r_{i j}, \varphi_{k}(i, j=1, \ldots, n+1 ; k=1, \ldots, n)$ and relations

$$
\begin{array}{clc}
r_{i j} r_{i k}= & r_{i k} r_{i j}, \quad r_{i k} r_{h k}=r_{h k} r_{i k} & \forall j<k, i<h \\
r_{i l} r_{j k}=r_{j k} r_{i l}, \quad & r_{i k} r_{j l}-r_{j l} r_{i k}=(0)^{1+\delta_{i k}+\delta_{j l}-\delta_{i l}-\delta_{j k}} r_{i l} r_{j k} & \forall i<j, k<l \\
r_{1,1} r_{2,2} \cdots r_{n+1, n+1}=1 & \\
0=r_{i i}-r_{i+1, i+1} & \forall i=1, \ldots, n
\end{array}
$$




$$
\begin{array}{crc}
\varphi_{i} r_{j k}-r_{j k} \varphi_{i}=0 & \forall j<i, k>i+1, \forall j>i+1, k<i \\
\varphi_{i} r_{j k}-r_{j k} \varphi_{i}=0 & \forall j<i, k<i \\
\varphi_{i} r_{j k}-r_{j k} \varphi_{i}=0 & \forall j>i+1, k>i+1 \\
\varphi_{i} r_{j i}-r_{j i} \varphi_{i}=-r_{i i} r_{j i} & \forall j<i \\
\varphi_{i} r_{j i}-r_{j i} \varphi_{i}=r_{j i} r_{i i} & \forall j>i+1 \\
\varphi_{i} r_{j, i+1}-r_{j, i+1} \varphi_{i}=r_{i+1, i+1} r_{j, i+1} & \forall j<i \\
\varphi_{i} r_{j, i+1}-r_{j, i+1} \varphi_{i}=-r_{j, i+1} r_{i+1, i+1} & \forall j<i \\
\varphi_{i} r_{i j}-r_{i j} \varphi_{i}=-r_{i i} r_{i j} & \forall j<i \\
\varphi_{i} r_{i j}-r_{i j} \varphi_{i}=r_{i j} r_{i i} & \forall j>i+1 \\
\varphi_{i} r_{i+1, j}-r_{i+1, j} \varphi_{i}=r_{i+1, i+1} r_{i+1, j} & \forall j<i \\
\varphi_{i} r_{i+1, j}-r_{i+1, j} \varphi_{i}=-r_{i+1, j} r_{i+1, i+1} & \forall j>i+1 \\
\varphi_{i} r_{i i}-r_{i i} \varphi_{i}=0 & \forall i \\
\varphi_{i} r_{i+1, i+1}-r_{i+1, i+1} \varphi_{i}=0 & \forall i \\
\varphi_{i} r_{i, i+1}-r_{i, i+1} \varphi_{i}=r_{i, i+1} r_{i i}+r_{i+1, i+1} r_{i, i+1} & \forall i \\
\varphi_{i} r_{i+1, i}-r_{i+1, i} \varphi_{i}=r_{i+1, i} r_{i i}+r_{i+1, i+1} r_{i+1, i} & \forall i \\
\varphi_{i} \varphi_{j}-\varphi_{j} \varphi_{i}=0 & \forall i, j
\end{array}
$$

Moreover, its Hopf structure is given by

$$
\begin{array}{cr}
\Delta\left(r_{i j}\right)=r_{i i} \otimes r_{i j}+r_{i j} \otimes r_{j j} & \forall i \neq j \\
\Delta\left(r_{i i}\right)=r_{i i} \otimes r_{i i} & \forall i \\
\Delta\left(\varphi_{i}\right)=r_{i i} \otimes \varphi_{i}+\varphi_{i} \otimes r_{i+1, i+1} & \forall i \\
S\left(r_{i j}\right)=-r_{i j} \cdot \prod_{\substack{k=1 \\
k \neq i, j}}^{n+1} r_{k, k} & \forall i, j \\
S\left(\varphi_{i}\right)=-r_{1,1} r_{2,2} \cdots r_{i-1, i-1} \varphi_{i} r_{i+2, i+2} \cdots r_{n+1, n+1} & \forall i \\
\varepsilon\left(r_{i j}\right)=\delta_{i j}, \quad \varepsilon\left(\varphi_{i}\right)=0 & \forall i, j .
\end{array}
$$

In particular we have that $r_{1,1}=r_{2,2}=\cdots=r_{n+1, n+1}$, hence $r_{i, i}^{n+1}=1$ for all $i$, whence $r_{i, i} \in k$ ( $k$ is algebraically closed); but then $\Delta\left(r_{i i}\right)=r_{i i} \otimes r_{i i}$ implies $r_{i i}=1$. Now relations $r_{i k} r_{j l}-r_{j l} r_{i k}=(0)^{1+\delta_{i k}+\delta_{j l}-\delta_{i l}-\delta_{j k}} r_{i l} r_{j k}(i<j, k<l)$ gives in particular $r_{i, j-1} r_{j-1, j}-r_{j-1, j} r_{i, j-1}=(0)^{\delta_{i, j-1}} r_{i, j} r_{j-1, j-1}(i<j)$, and similarly $r_{i, j+1} r_{j+1, j}-$ $r_{j+1, j} r_{i, j+1}=(0)^{\delta_{i, j+1}} r_{i, j} r_{j+1, j+1}(i<j)$, whence we deduce that the elements $r_{i, i+1}$, $r_{i+1, i}(i=1, \ldots, n)$ together with the $\varphi_{j}$ 's are enough to generate $\widetilde{F}_{1}^{Q}[S L(n+1)]$.

Now from the relations above one finds that for the generators $r_{i, i+1}, \varphi_{i}, r_{i+1, i}(i=$ $1, \ldots, n)$ exactly the same relations hold than we have in $\S 1$ for the generators $-\mathrm{f}_{i}, \mathrm{~h}_{i}, \mathrm{e}_{i}$, of $U(\mathfrak{h})$ : therefore $\widetilde{F}_{1}^{Q}[S L(n+1)]$ and $U(\mathfrak{h})$, having the same presentation, are isomorphic as $k$-algebras. Nevertheless, the formulae for the values of Hopf operations (of $\widetilde{F}_{1}^{Q}[S L(n+1)]$ ) on the generators $r_{i, i+1}, \varphi_{i}, r_{i+1, i}$ are exactly the same — when taking into account that 
$r_{i i}=1$ for all $i$ - than similar formulae for the generators $-\mathrm{f}_{i}, \mathrm{~h}_{i}, \mathrm{e}_{i}$, of $U(\mathfrak{h})$ : thus the $k$-algebra isomorphism $\Phi: \widetilde{F}_{1}^{Q}[S L(n+1)] \longrightarrow U(\mathfrak{h})$ given by

$$
\Phi: \quad r_{i, i+1} \mapsto-\mathrm{f}_{i}, \quad \varphi_{i} \mapsto \mathrm{h}_{i}, \quad r_{i+1, i} \mapsto+\mathrm{e}_{i}
$$

is even one of Hopf algebras. In particular, the comultiplication of $\widetilde{F}_{1}^{Q}[S L(n+1)]$ is cocommutative: hence a Poisson cobracket $\delta: \widetilde{F}_{1}^{Q}[S L(n+1)] \longrightarrow \widetilde{F}_{1}^{Q}[S L(n+1)] \otimes \widetilde{F}_{1}^{Q}[S L(n+1)]$ is canonically defined by $\delta\left(\left.x\right|_{q=1}\right):=\left.\frac{\left(\Delta-\Delta^{o p}\right)(x)}{q-1}\right|_{q=1}$.

In order to compare the latter Poisson cobracket with the one on $U(\mathfrak{h})$ given from scratch, we have to unravel the preimage in $\widetilde{F}_{1}^{Q}[S L(n+1)]$ of root vectors in $U(\mathfrak{h})$.

We already saw that $r_{i i}=1(i=1, \ldots, n+1)$ in $\widetilde{F}_{1}^{Q}[S L(n+1)]$; from this fact and the relations $r_{i k} r_{j l}-r_{j l} r_{i k}=(0)^{1+\delta_{i k}+\delta_{j l}-\delta_{i l}-\delta_{j k}} r_{i l} r_{j k}(i<j, k<l)$, for $j=k$ one gets

$$
r_{i l}=+\left[r_{i j}, r_{j l}\right] \quad \forall i<j<l,
$$

and similarly for $i=l$

$$
r_{j k}=-\left[r_{j i}, r_{i k}\right] \quad \forall j>i>k
$$

in particular

$$
\begin{array}{ll}
r_{i j}=+\left[r_{i, j-1}, r_{j-1, j}\right]=-\left[r_{j-1, j}, r_{i, j-1}\right] & \forall i<j-1, \\
r_{j i}=-\left[r_{j, j-1}, r_{j-1, i}\right]=+\left[r_{j-1, i}, r_{j, j-1}\right] & \forall j>i+1 .
\end{array}
$$

Comparing (4.2) and (1.1), by a simple induction one gets from (4.1) that

$$
\Phi\left(r_{i j}\right)=(-1)^{j-i} \mathrm{f}_{j i}, \quad \Phi\left(r_{j i}\right)=(-1)^{j-i-1} \mathrm{e}_{i j}, \quad \forall i<j .
$$

Now, a straightforward computation gives

$$
\begin{gathered}
\delta\left(r_{i, i+1}\right)=\varphi_{i} \otimes r_{i, i+1}-r_{i, i+1} \otimes \varphi_{i}+2 \cdot \sum_{j=1}^{n+1} \widehat{i} \widehat{i+1}\left(r_{i, j} \otimes r_{j, i+1}-r_{j, i+1} \otimes r_{i, j}\right) \\
\delta\left(\varphi_{i}\right)=4 \cdot\left(\sum_{j=1}^{n+1}\left(r_{i, j} \otimes r_{j, i}-r_{j, i} \otimes r_{i, j}\right)-\sum_{j=1}^{n+1}\left(r_{i+1, j} \otimes r_{j, i+1}-r_{j, i+1} \otimes r_{i+1, j}\right)\right) \\
\delta\left(r_{i+1, i}\right)=r_{i+1, i} \otimes \varphi_{i}-\varphi_{i} \otimes r_{i+1, i}+2 \cdot \sum_{j=1}^{n+1} \widehat{i} \widehat{i+1}\left(r_{i+1, j} \otimes r_{j, i}-r_{j, i} \otimes r_{i+1, j}\right)
\end{gathered}
$$

(for all $i=1, \ldots, n$ ), where a superscript $\widehat{h}$ means that the index $h$ must be discarded; then it is a simple task of rewriting (using (4.3)) to see that these formulae correspond - via $\Phi$ - to the analogous ones for $U(\mathfrak{h})$. Thus the isomorphism $\Phi: \widetilde{F}_{1}^{Q}[S L(n+1)] \longrightarrow U(\mathfrak{h})$ above is one of Poisson Hopf coalgebras; so we have proved the claim for $\widetilde{F}_{1}^{Q}[S L(n+1)]$. 
As for the other two algebras, we shall shortly conclude relying on the first one. In fact $\widetilde{F}_{1}^{P}[S L(n+1)]$ and $\widetilde{F}_{1}[S L(n+1)]$ differ from $\widetilde{F}_{1}^{Q}[S L(n+1)]$ only for "toral" generators: the $\psi_{i}$ 's, resp. the $\chi_{i}$ 's, instead of the $\varphi_{i}$ 's. Now, definitions give at once

$$
\psi_{i}=\chi_{1}+r_{1,1} \chi_{2}+r_{1,1} r_{2,2} \chi_{3}+\cdots+r_{1,1} r_{2,2} \cdots r_{i-1, i-1} \chi_{i} \quad \forall i=1, \ldots, n+1
$$

so that, since $r_{j, j} \equiv 1 \bmod (q-1)$, we have that $\left\{\psi_{1}, \ldots, \psi_{n+1}\right\}$ modulo $(q-1)$ and $\left\{\chi_{1}, \ldots, \chi_{n+1}\right\}$ modulo $(q-1)$ span the same $k$-vector space, whence

$$
\widetilde{F}_{1}^{P}[S L(n+1)]=\widetilde{F}_{1}[S L(n+1)] .
$$

Furthermore, definitions give also

$$
\varphi_{i}=\chi_{i}-\chi_{i+1} \quad \forall i=1, \ldots, n
$$

hence the $k$-span of $\left\{\varphi_{1}, \ldots, \varphi_{n+1}\right\}$ modulo $(q-1)$ is contained in the $k$-span of $\left\{\chi_{1}, \ldots\right.$, $\left.\chi_{n+1}\right\}$ modulo $(q-1)$; moreover, the relation

$$
\begin{aligned}
\sum_{i=1}^{n+1} r_{1,1} r_{2,2} & \cdots r_{i-1, i-1} \chi_{i}= \\
& =\sum_{\sigma \in S_{n+1} \backslash\{1\}}(-q)^{l(\sigma)}(q-1)^{e(\sigma)-1}\left(1+q^{-1}\right)^{e(\sigma)} r_{1, \sigma(1)} r_{2, \sigma(2)} \cdots r_{n+1, \sigma(n+1)}
\end{aligned}
$$

for $q=1$ turns into

$$
\chi_{1}+\cdots+\chi_{n+1}=0 ;
$$

thus the $k$-span of $\left\{\varphi_{1}, \ldots, \varphi_{n+1}\right\}$ modulo $(q-1)$ and the $k$-span of $\left\{\chi_{1}, \ldots, \chi_{n+1}\right\}$ modulo $(q-1)$ have both dimension $n$, hence they coincide. We conclude that

$$
\widetilde{F}_{1}^{Q}[S L(n+1)]=\widetilde{F}_{1}[S L(n+1)]=\widetilde{F}_{1}^{P}[S L(n+1)]
$$

whence the claim.

\section{$\S \mathbf{5} \quad \widetilde{F}_{q}^{M}[S L(n+1)]$ as approximation of $\mathcal{F}^{M}[S L(n+1)]$}

5.1 Motivations. To explain the definitions of the integer forms of $\S 4$ some comments are in order. We resume the analysis in [Ga], using the same notation, and make it more explicit for $G=S L(n+1)$.

Given the quantized universal enveloping algebra $U_{q}^{P}(\mathfrak{s l}(n+1))$, resp. $U_{q}^{Q}(\mathfrak{s l}(n+1))$, there exists a $k\left[q, q^{-1}\right]$-integer form (as Hopf algebra) $\mathcal{U}^{P}(\mathfrak{s l}(n+1))$, resp. $\mathcal{U}^{P}(\mathfrak{s l}(n+1))$ (cf. [Ga], §3.4); then we define (cf. [Ga], §4.3)

$$
\begin{aligned}
& \mathcal{F}^{Q}[S L(n+1)]:=\left\{f \in F_{q}^{Q}[S L(n+1)] \mid\left\langle f, \mathcal{U}^{P}(\mathfrak{s l}(n+1))\right\rangle \subseteq k\left[q, q^{-1}\right]\right\} \\
& \mathcal{F}^{P}[S L(n+1)]:=\left\{f \in F_{q}^{P}[S L(n+1)] \mid\left\langle f, \mathcal{U}^{Q}(\mathfrak{s l}(n+1))\right\rangle \subseteq k\left[q, q^{-1}\right]\right\}
\end{aligned}
$$


From the very definition of $\mathcal{U}^{P}$, one sees that $\widetilde{F}_{q}^{Q}[S L(n+1)] \subseteq \mathcal{F}^{Q}[S L(n+1)]$, and similarly, $\widetilde{F}_{q}^{P}[S L(n+1)] \subseteq \mathcal{F}^{P}[S L(n+1)]$. But even more, we shall prove in this section that $\widetilde{F}_{q}^{Q}[S L(n+1)]$, resp. $\widetilde{F}_{q}^{P}[S L(n+1)]$, is a "good enough approximation" of $\mathcal{F}^{Q}[S L(n+$ 1)], resp. $\mathcal{F}^{P}[S L(n+1)]$, in the sense that they have the same specialization at $q=1$.

One of the main points in $[\mathrm{Ga}]$ is the construction of a (topological) Hopf algebra $U_{q}^{P}(\mathfrak{h})$, with a $k\left[q, q^{-1}\right]$-integer form $\mathfrak{U}^{P}(\mathfrak{h})$ which specializes to $U(\mathfrak{h})$ for $q \rightarrow 1$. The link with quantum function algebras is the existence of an embedding of (topological) Hopf algebras

$$
\xi_{P}: F_{q}^{P}[S L(n+1)] \smile U_{q}^{P}(\mathfrak{h}) \text {; }
$$

via this embedding one has $\xi_{P}\left(\mathcal{F}^{P}[S L(n+1)]\right) \subseteq \mathfrak{U}^{P}(\mathfrak{h})$. In addition, one has also $\left.\xi_{P}\left(\mathcal{F}^{P}[S L(n+1)]\right)\right|_{q=1}=\left.\mathfrak{U}^{P}(\mathfrak{h})\right|_{q=1}$, so that $\mathfrak{U}^{P}(\mathfrak{h}) \stackrel{q \rightarrow 1}{\longrightarrow} U(\mathfrak{h})$ implies

$$
\mathcal{F}^{P}[S L(n+1)] \stackrel{q \rightarrow 1}{\longrightarrow} U(\mathfrak{h}) .
$$

The embedding $\xi_{P}: F_{q}^{P}[S L(n+1)] \hookrightarrow U_{q}^{P}(\mathfrak{h})$ is the composition of an embedding

$$
\mu_{P}: F_{q}^{P}[G] \stackrel{\Delta}{\longrightarrow} F_{q}^{P}[G] \otimes F_{q}^{P}[G] \stackrel{\rho_{+} \otimes \rho_{-}}{\longrightarrow} F_{q}^{P}\left[B_{+}\right] \otimes F_{q}^{P}\left[B_{-}\right] \stackrel{\vartheta_{+} \otimes \vartheta_{-}}{\longrightarrow} U_{q}^{P}\left(\mathfrak{b}_{-}\right)_{o p} \otimes U_{q}^{P}\left(\mathfrak{b}_{+}\right)_{o p}
$$

(where $G=S L(n+1)$, and $B_{ \pm}$and $\mathfrak{b}_{ \pm}$denotes as usual Borel subgroups and Borel subalgebras) and an isomorphism $\nu_{P}^{-1}$ of a suitable subalgebra of $U_{q}^{P}\left(\mathfrak{b}_{-}\right)_{o p} \otimes U_{q}^{P}\left(\mathfrak{b}_{+}\right)_{o p}$ (containing $\left.\mu_{P}\left(F_{q}^{P}[S L(n+1)]\right)\right)$ with $U_{q}^{P}(\mathfrak{h})$; hereafter, $H_{o p}$ will denote the (unique) Hopf algebra with the same structure of $H$ but for comultiplication, which is turned into the opposite one. Everything holds as well with $P$ and $Q$ exchanging their roles; on the other hand, since by definition is $F_{q}^{Q}[S L(n+1)] \subseteq F_{q}^{P}[S L(n+1)], U_{q}^{Q}(\mathfrak{h}) \subseteq U_{q}^{P}(\mathfrak{h})$, and $\xi_{Q}=\left.\xi_{P}\right|_{F_{q}^{Q}[S L(n+1)]}, \mu_{Q}=\left.\mu_{P}\right|_{F_{q}^{Q}[S L(n+1)]}$, it will be enough to study $\mu_{P}$. To this end, we have to revisit the definition of $U_{q}^{M}(\mathfrak{s l}(n+1))$ and its quantum Borel subalgebras $U_{q}^{M}\left(\mathfrak{b}_{ \pm}\right)(M=Q, P)$, and the construction of quantum root vectors: this will be done in next sections. Here we recall the definition of $F_{q}^{P}\left[B_{+}\right]$and $F_{q}^{P}\left[B_{-}\right]$and the canonical epimorphisms $\rho_{+}$and $\rho_{-}$.

$F_{q}^{P}\left[B_{+}\right]$, resp. $F_{q}^{P}\left[B_{-}\right]$, is the unital associative $k(q)$-algebra generated by $\left\{\rho_{i j} \mid i, j=\right.$ $1, \ldots, n+1 ; i \leq j\}$, resp. by $\left\{\rho_{i j} \mid i, j=1, \ldots, n+1 ; i \geq j\right\}$, with relations

$$
\begin{array}{clrl}
\rho_{i j} \rho_{i k}=q \rho_{i k} \rho_{i j}, \quad \rho_{i k} \rho_{h k} & =q \rho_{h k} \rho_{i k} & & \forall j<k, i<h \\
\rho_{i l} \rho_{j k}=\rho_{j k} \rho_{i l}, \quad \rho_{i k} \rho_{j l}-\rho_{j l} \rho_{i k} & =\left(q-q^{-1}\right) \rho_{i l} \rho_{j k} & \forall i<j, k<l \\
\rho_{1,1} \rho_{2,2} \cdots \rho_{n+1, n+1}=1 & &
\end{array}
$$

for either algebras. These are Hopf algebras too, with comultiplication given by

$$
\begin{array}{lll}
\Delta\left(\rho_{i j}\right)=\sum_{k=i}^{n} \rho_{i k} \otimes \rho_{k j} & \forall i, j & \text { for } F_{q}^{P}\left[B_{+}\right], \\
\Delta\left(\rho_{i j}\right)=\sum_{k=1}^{j} \rho_{i k} \otimes \rho_{k j} & \forall i, j & \text { for } F_{q}^{P}\left[B_{-}\right],
\end{array}
$$


counit by

$$
\epsilon\left(\rho_{i j}\right)=\delta_{i j} \quad \forall i, j
$$

for either algebras, and antipode by

$$
\begin{array}{lll}
S\left(\rho_{i j}\right)=(-q)^{j-i} \operatorname{det}_{q}^{+}\left(\left(\rho_{h k}\right)_{\substack{k \neq i \\
h \neq j}}\right) & \forall i, j \quad \text { for } F_{q}^{P}\left[B_{+}\right] \\
S\left(\rho_{i j}\right)=(-q)^{j-i} \operatorname{det}_{q}^{-}\left(\left(\rho_{h k}\right)_{h \neq j}^{k \neq i}\right) & \forall i, j \quad \text { for } F_{q}^{P}\left[B_{-}\right]
\end{array}
$$

where $d e t_{q}^{+}$, resp. $d e t_{q}^{-}$, is the expression that one gets simply by setting $\rho_{i j} \equiv 0$ for all $i>j$, resp. $i<j$, in $\operatorname{det}_{q}$.

By the very definitions, two Hopf algebra epimorphisms exist

$$
\rho_{+}: F_{q}^{P}[S L(n+1)] \longrightarrow F_{q}^{P}\left[B_{+}\right], \quad \rho_{-}: F_{q}^{P}[S L(n+1)] \longrightarrow F_{q}^{P}\left[B_{-}\right],
$$

given by

$$
\begin{array}{llll}
\rho_{+}: & \rho_{i j} \mapsto \rho_{i j} & \forall i \leq j, & \rho_{i j} \mapsto 0 \forall i>j \\
\rho_{-}: & \rho_{i j} \mapsto \rho_{i j} & \forall i \geq j, & \rho_{i j} \mapsto 0 \forall i<j
\end{array}
$$

5.2 The quantum algebras $U_{q}(\mathfrak{g l}(n+1)), U_{q}(\mathfrak{s l}(n+1))$, and $U_{q}^{P}\left(\mathfrak{b}_{ \pm}\right)$. We recall (cf. for instance $[\mathrm{GL}])$ the definition of the quantized universal enveloping algebra $U_{q}(\mathfrak{g l}(n+$ $1))$ : it is the associative algebra with 1 over $k(q)$ with generators

$$
F_{1}, F_{2}, \ldots, F_{n}, G_{1}^{ \pm 1}, G_{2}^{ \pm 1}, \ldots, G_{n}^{ \pm 1}, G_{n+1}^{ \pm 1}, E_{1}, E_{2}, \ldots, E_{n}
$$

and relations

$$
\begin{array}{cc}
G_{i} G_{i}^{-1}=1=G_{i}^{-1} G_{i}, \quad G_{i}^{ \pm 1} G_{j}^{ \pm 1}=G_{j}^{ \pm 1} G_{i}^{ \pm 1} & \forall i, j \\
G_{i} F_{j} G_{i}^{-1}=q^{\delta_{i, j+1}-\delta_{i, j}} F_{j}, \quad G_{i} E_{j} G_{i}^{-1}=q^{\delta_{i, j}-\delta_{i, j+1}} E_{j} & \forall i, j \\
E_{i} F_{j}-F_{j} E_{i}=\delta_{i, j} \frac{G_{i} G_{i+1}^{-1}-G_{i}^{-1} G_{i+1}}{q-q^{-1}} & \forall i, j \\
E_{i} E_{j}=E_{j} E_{i}, \quad F_{i} F_{j}=F_{j} F_{i} & \forall i, j:|i-j|>1 \\
E_{i}^{2} E_{j}-\left(q+q^{-1}\right) E_{i} E_{j} E_{i}+E_{j} E_{i}^{2}=0 & \forall i, j:|i-j|=1 \\
F_{i}^{2} F_{j}-\left(q+q^{-1}\right) F_{i} F_{j} F_{i}+F_{j} F_{i}^{2}=0 & \forall i, j:|i-j|=1 .
\end{array}
$$

Moreover, $U_{q}(\mathfrak{g l}(n+1))$ has a Hopf algebra structure, given by

$$
\begin{array}{cccc}
\Delta\left(F_{i}\right)=F_{i} \otimes G_{i}^{-1} G_{i+1}+1 \otimes F_{i}, & S\left(F_{i}\right)=-F_{i} G_{i} G_{i+1}^{-1}, & \epsilon\left(F_{i}\right)=0 & \forall i \\
\Delta\left(G_{i}^{ \pm 1}\right)=G_{i}^{ \pm 1} \otimes G_{i}^{ \pm 1}, & S\left(G_{i}^{ \pm 1}\right)=G_{i}^{\mp 1}, & \epsilon\left(G_{i}^{ \pm 1}\right)=1 & \forall i \\
\Delta\left(E_{i}\right)=E_{i} \otimes 1+G_{i} G_{i+1}^{-1} \otimes E_{i}, & S\left(E_{i}\right)=-G_{i}^{-1} G_{i+1} E_{i}, & \epsilon\left(E_{i}\right)=0 & \forall i .
\end{array}
$$

The algebras $U_{q}^{P}(\mathfrak{s l}(n+1))$ and $U_{q}^{Q}(\mathfrak{s l}(n+1))$ - defined as in [Ga], 33 - can be realized as Hopf subalgebras or quotients of $U_{q}(\mathfrak{g l}(n+1))$. Namely, define elements

$$
L_{i}:=G_{1} \cdots G_{i}, \quad L_{i}^{-1}:=G_{1}^{-1} \cdots G_{i}^{-1}, \quad K_{i}:=G_{i} G_{i+1}^{-1}, \quad K_{i}^{-1}:=G_{i}^{-1} G_{i+1}
$$


for all $i=1, \ldots, n$. Then $L_{n+1}$ is a central element of $U_{q}(\mathfrak{g l}(n+1))$, and $U_{q}^{P}(\mathfrak{s l}(n+1))$ is (isomorphic to) the subalgebra of $U_{q}(\mathfrak{g l}(n+1))$ generated by $\left\{F_{1}, \ldots, F_{n}, L_{1}^{ \pm 1}, \ldots, L_{n}^{ \pm 1}\right.$, $\left.E_{1}, \ldots, E_{n}\right\}$ - this corresponds to $\mathfrak{s l}(n+1) \hookrightarrow \mathfrak{g l}(n+1)$ - and to the quotient of $U_{q}(\mathfrak{g l}(n+1))$ modulo the ideal (which is a Hopf ideal) generated by $\left(L_{n+1}-1\right)$ - which corresponds to $\mathfrak{s l}(n+1) \cong \mathfrak{g l}(n+1) /\left(I_{n+1}\right)=\mathfrak{g l}(n+1) / Z(\mathfrak{g l}(n+1))$. Similarly, the algebra $U_{q}^{Q}(\mathfrak{s l}(n+1))$ is (isomorphic to) the subalgebra of $U_{q}(\mathfrak{g l}(n+1))$ generated by $\left\{F_{1}, \ldots, F_{n}, K_{1}^{ \pm 1}, \ldots, K_{n}^{ \pm 1}, E_{1}, \ldots, E_{n}\right\}$ - this again corresponds to $\mathfrak{s l}(n+1) \hookrightarrow \mathfrak{g l}(n+1)$.

A last word about "toral elements" $G_{i}, L_{i}, K_{i}$. In the "classical" framework we have toral elements $h_{i}=h_{\alpha_{i}}$ in the (diagonal) Cartan subalgebra of $\mathfrak{s l}(n+1)(\subseteq \mathfrak{g l}(n+1))$, given by $h_{i}=M_{i, i}-M_{i+1, i+1}$ (where $M_{r, s}$ denotes a square matrix of size $n+1$ as in $\S 2)$; similarly, letting $\ell_{i}=M_{11}+M_{22}+\cdots+M_{i i}$, we have $h_{i}=-\ell_{i-1}+2 \ell_{i}-$ $\ell_{i+1}$. Now, $G_{i}$ is the $q$-analogue of $M_{i, i}$ - in fact, on the standard representation it acts exactly as $\exp \left(h M_{i, i}\right)$, where $h:=\log (q)$ - therefore $K_{i}:=G_{i} G_{i+1}^{-1}=\exp \left(h M_{i, i}\right)$. $\exp \left(h M_{i+1, i+1}\right)^{-1}=\exp \left(h\left(M_{i, i}-M_{i+1, i+1}\right)\right)=\exp \left(h \cdot h_{i}\right)$ is exactly the $q$-analogue of $h_{i}$; similarly, $L_{i}$ is the $q$-analogue of $\ell_{i}$.

As for quantum Borel subalgebras, we recall that $U_{q}^{P}\left(\mathfrak{b}_{+}\right)$, resp. $U_{q}^{P}\left(\mathfrak{b}_{-}\right)$, is - by definition — the subalgebra of $U_{q}^{P}(\mathfrak{s l}(n+1))$ generated by $\left\{L_{1}, \ldots, L_{n}\right\} \cup\left\{E_{1}, \ldots, E_{n}\right\}$, resp. by $\left\{L_{1}, \ldots, L_{n}\right\} \cup\left\{F_{1}, \ldots, F_{n}\right\}$; similar definitions occur for $U_{q}^{Q}\left(\mathfrak{b}_{ \pm}\right)$, with $K_{i}$ 's instead of $L_{i}$ 's. All these are in fact Hopf subalgebras.

Finally, there exist Hopf algebra isomorphisms

$$
\vartheta_{+}: F_{q}^{P}\left[B_{+}\right] \stackrel{\cong}{\longrightarrow} U_{q}^{P}\left(\mathfrak{b}_{-}\right)_{o p}, \quad \vartheta_{-}: F_{q}^{P}\left[B_{-}\right] \stackrel{\cong}{\longrightarrow} U_{q}^{P}\left(\mathfrak{b}_{+}\right)_{o p}
$$

which are uniquely determined by

$$
\begin{array}{cll}
\vartheta_{+}\left(\rho_{i i}\right):=L_{i-1} L_{i}^{-1}=G_{i}^{-1}, \quad \vartheta_{+}\left(\rho_{i, i+1}\right):=-\bar{F}_{i} L_{i} L_{i+1}^{-1}=-\bar{F}_{i} G_{i+1}^{-1}, & \forall i \\
\vartheta_{-}\left(\rho_{i i}\right):=L_{i-1}^{-1} L_{i}=G_{i}, \quad \vartheta_{-}\left(\rho_{i+1, i}\right):=+L_{i}^{-1} L_{i+1} \bar{E}_{i}=+G_{i+1} \bar{E}_{i}, & \forall i
\end{array}
$$

(here we set $\left.L_{0}:=1, L_{n+1}:=1\right)$, where $\bar{F}_{i}:=\left(q-q^{-1}\right) F_{i}, \bar{E}_{i}:=\left(q-q^{-1}\right) E_{i}$.

5.3 Quantum root vectors. Quantum root vectors are essential in [Ga]: according to a general recipe provided by Lusztig, they are constructed by means of braid group operators $T_{i}(i=1, \ldots, n)$, which in our case are given by (with the normalization of [Ga])

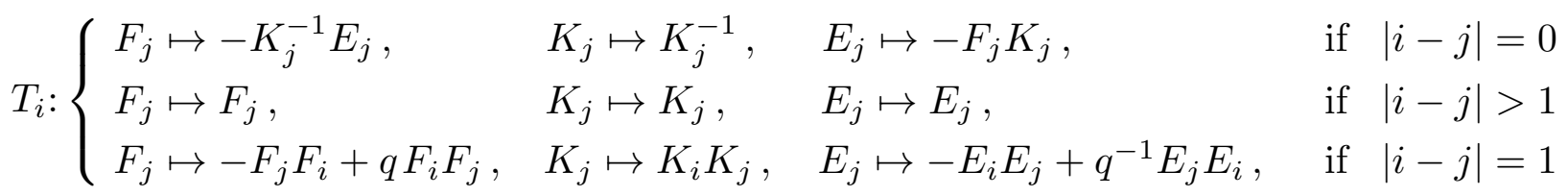

Thus letting $[x, y]_{q}:=x y-q y x$ be the $q$-bracket of $x$ and $y$ we have $T_{i}\left(F_{j}\right)=q\left[F_{i}, F_{j}\right]_{q^{-1}}=-\left[F_{j}, F_{i}\right]_{q}, T_{i}\left(E_{j}\right)=-\left[E_{i}, E_{j}\right]_{q^{-1}}=q^{-1}\left[E_{j}, E_{i}\right]_{q}$ for $|i-j|=1$.

Consider now the case $G=S L(n+1), \mathfrak{g}=\mathfrak{s l}(n+1)$. We want to compare Lusztig's construction of quantum root vectors with another one (which is used, for instance, in [Ji] and in $[\mathrm{Ta}])$. 
In the Lie algebra $\mathfrak{s l}(n+1)$ we have matrices $M_{i j}(i, j \in\{1,2, \ldots, n\}, i<j)$ such that

$$
M_{i, i+1}=e_{i}, \quad\left[M_{i k}, M_{k j}\right]=M_{i j}, \quad \forall i<k<j
$$

where $e_{i}$ denotes the $i$-th Chevalley generator of the positive part of $\mathfrak{s l}(n+1)$, the Lie subalgebra $\mathfrak{n}_{+}$of strictly upper triangular matrices. Moreover, these matrices are root vectors, i. e. weight vectors - for the adjoint action — with roots as weights: namely, $M_{i j}$ has weight the positive root $\alpha(i, j):=\varepsilon_{i}-\varepsilon_{j}$, where $\varepsilon_{k}$ denotes the $(k, k)$-th coordinate function on matrices. Notice that the same is true for $-\left[M_{i k}, M_{k j}\right]=-M_{i j}$. Then the level of $M_{i j}$, defined to be $j-i$, is the height of the root $\alpha(i, j)$. A similar situation occurs for the subalgebra $\mathfrak{n}_{-}$of strictly lower triangular matrices, with elements $M_{i j}(i>j)$ as root vectors of weight the negative roots $\alpha(i, j)$ of height $j-i$. In particular, $\left\{M_{i j} \mid i<j\right\}$ is a basis of $\mathfrak{n}_{+}$, and $\left\{M_{i j} \mid i>j\right\}$ is a basis of $\mathfrak{n}_{-}$.

The situation described above can be quantized. In fact in the standard representation of $U_{q}^{P}(\mathfrak{s l}(n+1))$ or $U_{q}^{Q}(\mathfrak{s l}(n+1))$ we still have $E_{i} \mapsto M_{i, i+1}$, i.e. $E_{i}$ acts as the matrix $M_{i, i+1}$; then we define (for all $i<j-1$ )

$$
\begin{array}{ll}
E_{i, i+1}:=E_{i}, & E_{i, j}:=-\left[E_{i, j-1}, E_{j-1, j}\right]_{q^{-1}}=q^{-1}\left[E_{j-1, j}, E_{i, j-1}\right]_{q} \\
F_{j+1, j}:=F_{i}, & F_{j, i}:=q\left[F_{j-1, i}, F_{j, j-1}\right]_{q^{-1}}=-\left[F_{j, j-1}, F_{j-1, i}\right]_{q}
\end{array}
$$

(notice the occurrence of the "-" sign, which does not appear in [Ji], nor in [Ta]); then remark also that in the standard representation of $U_{q}^{P}(\mathfrak{s l}(n+1))$ or $U_{q}^{Q}(\mathfrak{s l}(n+1))$ we have $E_{i j} \mapsto(-1)^{j-i-1} M_{i j}$ and $F_{j i} \mapsto(-1)^{j-i-1} M_{j i}$, for all $i<j$.

Now look at roots, for instance positive ones: they form the set

$$
R^{+}:=\{\alpha(i, j) \mid i, j=1, \ldots, n+1 ; i<j\}
$$

if we set $n(i, j):=i-j+\sum_{h=0}^{i-1}(n-h)$, we obtain a total ordering of $R^{+}$by

$$
\alpha(i, j) \preceq \alpha(h, k) \Longleftrightarrow n(i, j) \leq n(h, k)
$$

so that $R^{+}=\left\{\alpha^{1}, \alpha^{2}, \ldots, \alpha^{N}\right\}$, with $\alpha(i, j)=: \alpha^{n(i, j)}, N:=\left(\begin{array}{c}n+1 \\ 2\end{array}\right)$.

The first key point is the following lemma, whose proof is trivial:

Lemma. The previously defined ordering of $R^{+}$is convex, that is

$$
\alpha, \beta, \alpha+\beta \in R^{+}, \alpha \preceq \beta \Longrightarrow \alpha \preceq \alpha+\beta \preceq \beta \text {. }
$$

By a theorem of Papi (cf. [Pa]) we know that every total ordering of $R^{+}$which is convex is associated to a unique minimal expression of $w_{0}=(n n-1 \ldots 321)$, the longest element of the Weyl group $S_{n}$ of $\mathfrak{s l}(n+1)$ : this means that, if $w_{0}=s_{i_{1}} s_{i_{2}} \cdots s_{i_{N-1}} s_{i_{N}}$ is a minimal expression of $w_{0}$, then the ordering of $R^{+}$is given by

$\alpha^{1}=\alpha_{i_{1}}, \alpha^{2}=s_{i_{1}}\left(\alpha_{i_{2}}\right), \ldots, \alpha^{k}=s_{i_{1}} s_{i_{2}} \cdots s_{i_{k-1}}\left(\alpha_{i_{k}}\right), \ldots, \alpha^{N}=s_{i_{1}} s_{i_{2}} \cdots s_{i_{N-1}}\left(\alpha_{i_{N}}\right)$. 
In the present situation we can also write down explicitely the minimal expression of $w_{0}$ afforded by the given ordering of $R^{+}$: it is

$$
w_{0}=s_{1} s_{2} s_{3} \cdots s_{n-1} s_{n} s_{1} s_{2} \cdots s_{n-1} s_{1} s_{2} \cdots s_{n-3} s_{1} s_{2} \cdots s_{4} s_{1} s_{2} s_{3} s_{1} s_{2} s_{1}
$$

Starting from any minimal expression of $w_{0}$, Lusztig constructs quantum root vectors via the formulae

$$
E_{\alpha^{k}}:=T_{w_{k-1}}\left(E_{i_{k}}\right)=T_{i_{1}} T_{i_{2}} \cdots T_{i_{k-1}}\left(E_{i_{k}}\right), \quad F_{\alpha^{k}}:=T_{w_{k-1}}\left(F_{i_{k}}\right)=T_{i_{1}} T_{i_{2}} \cdots T_{i_{k-1}}\left(F_{i_{k}}\right)
$$

where $w_{0}=s_{i_{1}} s_{i_{2}} \cdots s_{i_{N}}$ is the given minimal expression, $w_{h}:=s_{i_{1}} s_{i_{2}} \cdots s_{i_{h}}$, and $\alpha^{k}=$ $w_{k-1}\left(\alpha_{i_{k}}\right)$ gives the associated convex ordering of $R^{+}$. In our case this construction gives quantum root vectors (for all $i<j$ )

$$
\begin{aligned}
E_{\alpha^{n(i, j)}} & :=T_{1} T_{2} \cdots T_{n} T_{1} T_{2} \cdots T_{n-1} T_{1} \cdots T_{n-i+1} T_{1} T_{2} \cdots T_{j-i-1}\left(E_{j-i}\right) \\
F_{\alpha^{n(i, j)}} & :=T_{1} T_{2} \cdots T_{n} T_{1} T_{2} \cdots T_{n-1} T_{1} \cdots T_{n-i+1} T_{1} T_{2} \cdots T_{j-i-1}\left(F_{j-i}\right)
\end{aligned}
$$

Theorem. For all $i, j=1,2, \ldots, n+1$ with $i<j$ we have

$$
E_{\alpha^{n(i, j)}}=E_{i j}, \quad F_{\alpha^{n(i, j)}}=F_{j i}
$$

Proof. We make the proof for the "E" case, the "F" case is the like.

We have two possibilities, $j=i+1$ and $j>i+1$. If $j=i+1$ we have

$$
\alpha^{n(i, j)}=s_{1} s_{2} \cdots s_{n} s_{1} s_{2} \cdots s_{n-i+2} s_{1} s_{2} \cdots s_{n-i+1}\left(\alpha_{1}\right)=s_{1} s_{2} \cdots s_{n} s_{1} s_{2} \cdots s_{n-i+2} s_{1} s_{2}\left(\alpha_{1}\right)
$$

because $s_{h}\left(\alpha_{k}\right)=\alpha_{k}$ for all $|h-k|>1$; since $s_{1} s_{2}\left(\alpha_{1}\right)=\alpha_{2}$, we get

$$
\begin{aligned}
& \alpha^{n(i, j)}=s_{1} s_{2} \cdots s_{n} s_{1} s_{2} \cdots s_{n-i+2}\left(\alpha_{2}\right)= \\
& =s_{1} s_{2} \cdots s_{n} s_{1} s_{2} \cdots s_{n-i+3} s_{1} s_{2} s_{3}\left(\alpha_{2}\right)=s_{1} s_{2} \cdots s_{n} s_{1} s_{2} \cdots s_{n-i+3}\left(\alpha_{3}\right) ;
\end{aligned}
$$

thus iterating we obtain

$$
\alpha^{n(i, j)}=\alpha_{i}
$$

then by Lusztig's work we know that $E_{\alpha^{n(i, j)}}=E_{\alpha_{i}}=E_{i}$; on the other hand, $j=i+1$ gives $E_{i, j}=E_{i, i+1}=E_{i}$ by definition, hence $E_{\alpha^{n(i, j)}}=E_{i, j}$, q.e.d.

If $j>i+1$ we apply the definitions to get

$$
\begin{gathered}
E_{\alpha^{n(i, j)}}:=T_{1} T_{2} \cdots T_{n} T_{1} T_{2} \cdots T_{n-1} T_{1} \cdots T_{n-i+1} T_{1} T_{2} \cdots T_{j-i-2} T_{j-i-1}\left(E_{j-i}\right) \\
=T_{1} T_{2} \cdots T_{n} T_{1} T_{2} \cdots T_{n-1} T_{1} \cdots T_{n-i+1} T_{1} T_{2} \cdots T_{j-i-2}\left(-\left[E_{j-i-1}, E_{j-i}\right]_{q^{-1}}\right)= \\
=-\left[T_{1} \cdots T_{n-i+1} T_{1} \cdots T_{j-i-2}\left(E_{j-i-1}\right), T_{1} \cdots T_{n-i+1} T_{1} \cdots T_{j-i-2}\left(E_{j-i}\right)\right]_{q^{-1}}= \\
\quad=-\left[E_{\alpha^{n(i, j-1)}}, T_{1} T_{2} \cdots T_{n} T_{1} T_{2} \cdots T_{n-i+1} T_{1} T_{2} \cdots T_{j-i-2}\left(E_{j-i}\right)\right]_{q^{-1}} ;
\end{gathered}
$$


but now we have

$$
\begin{array}{r}
T_{1} T_{2} \cdots T_{n} T_{1} T_{2} \cdots T_{n-i+2} \cdots T_{1} T_{2} \cdots T_{n-i+1} T_{1} T_{2} \cdots T_{j-i-2}\left(E_{j-i}\right)= \\
=T_{1} T_{2} \cdots T_{n} T_{1} T_{2} \cdots T_{n-i+2} T_{1} T_{2} \cdots T_{j-i-1} T_{j-i} T_{j-i+1}\left(E_{j-i}\right)= \\
\quad=T_{1} T_{2} \cdots T_{n} T_{1} T_{2} \cdots T_{n-i+2} T_{1} T_{2} \cdots T_{j-i-1}\left(E_{j-i+1}\right)
\end{array}
$$

for $T_{k} T_{k+1}\left(E_{k}\right)=E_{k+1}$ for all $k$; then a simple iteration gives

$$
T_{1} T_{2} \cdots T_{n} T_{1} T_{2} \cdots T_{n-i+2} \cdots T_{1} T_{2} \cdots T_{n-i+1} T_{1} T_{2} \cdots T_{j-i-2}\left(E_{j-i}\right)=E_{j-1} .
$$

Therefore we have

$$
E_{\alpha^{n(i, j)}}=-\left[E_{\alpha^{n(i, j-1)}}, E_{j-1}\right]_{q^{-1}} ;
$$

since $(j-1)-i<j-i$, we can use induction on the height of roots and assume $E_{\alpha^{n(i, j-1)}}=E_{i, j-1}$; on the other hand, $E_{j-1}=E_{j-1, j}$, by definition; thus we find $E_{\alpha^{n(i, j)}}=-\left[E_{i, j-1}, E_{j-1, j}\right]_{q^{-1}}$, and - by definition again - the right-hand-side term is nothing but $E_{i, j}$. The claim follows.

5.4 The embedding $\mu_{M}: F_{q}^{P}[S L(n+1)] \longrightarrow U_{q}^{P}\left(\mathfrak{b}_{-}\right)_{o p} \otimes U_{q}^{P}\left(\mathfrak{b}_{+}\right)_{o p}$ and specialization results. We are now ready to go on with the analysis of the embedding

$$
\mu_{P}: F_{q}^{P}[G] \stackrel{\Delta}{\longrightarrow} F_{q}^{P}[G] \otimes F_{q}^{P}[G] \stackrel{\rho_{+} \otimes \rho_{-}}{\longrightarrow} F_{q}^{P}\left[B_{+}\right] \otimes F_{q}^{P}\left[B_{-}\right] \stackrel{\vartheta_{+} \otimes \vartheta_{-}}{\longrightarrow} U_{q}^{P}\left(\mathfrak{b}_{-}\right)_{o p} \otimes U_{q}^{P}\left(\mathfrak{b}_{+}\right)_{o p}
$$

$(G=S L(n+1))$ : here $\Delta$ is the comultiplication of the Hopf algebra $F_{q}^{M}[S L(n+1)]$, $\rho_{ \pm}: F_{q}^{M}[S L(n+1)] \longrightarrow F_{q}^{M}\left[B_{ \pm}\right]$are the natural epimorphisms of $\S 5.1$, and the maps $\vartheta_{ \pm}: F_{q}^{M}\left[B_{ \pm}\right] \stackrel{\cong}{\longrightarrow} U_{q}^{M}\left(\mathfrak{b}_{\mp}\right)_{o p}$ are the isomorphisms given in $\S 5.2$.

To begin with, we go back to the study of $\widetilde{F}_{q}^{Q}[S L(n+1)]$ : lifting the identities $(4.2)$ gives relations (inside $\widetilde{F}_{q}^{Q}[S L(n+1)]$ )

$$
\begin{aligned}
r_{i j}=+\left[r_{i, j-1}, r_{j-1, j}\right]+\mathcal{O}(q-1)=-\left[r_{j-1, j}, r_{i, j-1}\right]+\mathcal{O}(q-1) & \forall i<j-1, \\
r_{j i}=-\left[r_{j, j-1}, r_{j-1, i}\right]+\mathcal{O}(q-1)=+\left[r_{j-1, i}, r_{j, j-1}\right]+\mathcal{O}(q-1) & \forall j>i+1 .
\end{aligned}
$$

Now look at the isomorphism $\vartheta_{+}: F_{q}^{P}\left[B_{+}\right] \stackrel{\cong}{\longrightarrow} U_{q}^{P}\left(\mathfrak{b}_{-}\right)_{o p}$; it maps $\rho_{i i}=r_{i i}$ onto $L_{i-1} L_{i}^{-1}=G_{i}^{-1}$, and $\rho_{i, i+1}$ onto $-\bar{F}_{i} L_{i} L_{i+1}^{-1}=-\bar{F}_{i}+G_{i+1}^{-1}$, hence $r_{i, i+1}$ onto $-F_{i} L_{i} L_{i+1}^{-1}$. But remark that $r_{i i}=1+\mathcal{O}(q-1)$, and so also $G_{i}=1+\mathcal{O}(q-1), K_{i}=1+\mathcal{O}(q-1)$, $L_{i}=1+\mathcal{O}(q-1)$, where the symbol $\mathcal{O}(q-1)$ denotes some element of $(q-1) \mathfrak{U}^{P}\left(\mathfrak{b}_{-}\right)$ (notations of $[\mathrm{Ga}]$ ): thus we have also $\vartheta_{+}\left(r_{i, i+1}\right)=-F_{i}+\mathcal{O}(q-1)$.

Therefore, using the first relation of (5.1), Theorem 5.3, and the fact that $[,]_{q} \equiv$ $[,]_{q^{-1}} \equiv[,] \bmod (q-1)$, by a simple iterating procedure we find that

$$
\vartheta_{+}\left(r_{i j}\right)=(-1)^{j-i} F_{j, i}+\mathcal{O}(q-1)=(-1)^{j-i} F_{\alpha^{n(i, j)}}+\mathcal{O}(q-1) \quad \forall i<j .
$$

Similarly, by the same arguments we can prove that

$$
\vartheta_{-}\left(r_{j i}\right)=(-1)^{j-i+1} E_{i, j}+\mathcal{O}(q-1)=(-1)^{j-i+1} E_{\alpha^{n(i, j)}}+\mathcal{O}(q-1) \quad \forall j>i .
$$


Now, from the definition of $\Delta$ and $\vartheta_{ \pm}$we get a description of $\mu_{P}\left(r_{i, i+1}\right)$ as follows:

$$
\begin{aligned}
& \mu_{P}\left(r_{i, i+1}\right)=\left(\vartheta_{+} \otimes \vartheta_{-}\right)\left(\left(\rho_{+} \otimes \rho_{-}\right)\left(\Delta\left(r_{i, i+1}\right)\right)\right)= \\
& =\left(\vartheta_{+} \otimes \vartheta_{-}\right)\left(\left(\rho_{+} \otimes \rho_{-}\right)\left(r_{i, i} \otimes r_{i, i+1}+r_{i, i+1} \otimes r_{i+1, i+1}+\left(q-q^{-1}\right) \sum_{\substack{k=1 \\
k \neq i, j}}^{n+1} r_{i, k} \otimes r_{k, i+1}\right)\right)= \\
& =\left(\vartheta_{+} \otimes \vartheta_{-}\right)\left(r_{i, i+1} \otimes r_{i+1, i+1}+\left(q-q^{-1}\right) \sum_{k=i+2}^{n+1} r_{i, k} \otimes r_{k, i+1}\right)= \\
& =-F_{i} G_{i+1}^{-1} \otimes G_{i+1}+\mathcal{O}(q-1)+\left(q-q^{-1}\right) \sum_{k=i+2}^{n+1} F_{\alpha(k, i)} \otimes E_{\alpha(i+1, k)}+\mathcal{O}\left((q-1)^{2}\right)= \\
& =-F_{i} G_{i+1}^{-1} \otimes G_{i+1}+\mathcal{O}(q-1)=-F_{i} \otimes 1+\mathcal{O}(q-1)
\end{aligned}
$$

where the symbol $\mathcal{O}(q-1)$ denotes some element of $(q-1) \mathfrak{U}^{P}\left(\mathfrak{b}_{-}\right) \otimes \mathfrak{U}^{P}\left(\mathfrak{b}_{+}\right)$(note that the last algebra is mapped by $\nu_{P}^{-1}$ into $\left.\mathfrak{U}^{P}(\mathfrak{h})\right)$; recalling from [Ga] the definition of $\nu_{P}^{-1}$ : $\mathfrak{U}^{P}\left(\mathfrak{b}_{-}\right) \otimes \mathfrak{U}^{P}\left(\mathfrak{b}_{+}\right) \longrightarrow \mathfrak{U}^{P}(\mathfrak{h})$, for $\xi_{P}=\mu_{P} \circ \nu_{P}^{-1}$ we find

$$
\xi_{P}\left(r_{i, i+1}\right)=-F_{i}+\mathcal{O}(q-1)
$$

and in general $\xi_{P}\left(r_{i, j}\right)=(-1)^{j-i} F_{j, i}+\mathcal{O}(q-1)$, for all $i<j$. A similar analysis yields

$$
\xi_{P}\left(r_{i+1, i}\right)=+E_{i}+\mathcal{O}(q-1)
$$

and in general $\xi_{P}\left(r_{j, i}\right)=(-1)^{j-i+1} E_{i, j}+\mathcal{O}(q-1)$, for all $j>i$, and also

$$
\xi_{P}\left(r_{i, i}\right)=G_{i}+\mathcal{O}\left((q-1)^{2}\right)=L_{i-1}^{-1} L_{i}+\mathcal{O}\left((q-1)^{2}\right) ;
$$

furthermore, the latter implies

$$
\begin{gathered}
\xi_{P}\left(\varphi_{i}\right)=\xi_{P}\left(\frac{r_{i, i}-r_{i+1, i+1}}{q-1}\right)=\frac{G_{i}-G_{i+1}}{q-1}+\mathcal{O}(q-1)= \\
=G_{i+1} \cdot \frac{G_{i} G_{i+1}^{-1}-1}{q-1}+\mathcal{O}(q-1)=G_{i+1} \cdot \frac{K_{i}-1}{q-1}+\mathcal{O}(q-1)= \\
=\frac{K_{i}-1}{q-1}+\mathcal{O}(q-1)=\left(\begin{array}{c}
K_{i} ; 0 \\
1
\end{array}\right)+\mathcal{O}(q-1), \\
\xi_{P}\left(\psi_{i}\right)=\xi_{P}\left(\frac{r_{1,1} \cdots r_{i, i}-1}{q-1}\right)=\frac{G_{1} \cdots G_{i}-1}{q-1}+\mathcal{O}(q-1)= \\
=\frac{L_{i}-1}{q-1}+\mathcal{O}(q-1)=\left(\begin{array}{c}
L_{i} ; 0 \\
1
\end{array}\right)+\mathcal{O}(q-1), \\
\xi_{P}\left(\chi_{i}\right)=\xi_{P}\left(\frac{r_{i, i}-1}{q-1}\right)=\frac{G_{i}-1}{q-1}+\mathcal{O}(q-1)=\left(\begin{array}{c}
G_{i} ; 0 \\
1
\end{array}\right)+\mathcal{O}(q-1) .
\end{gathered}
$$


Remark: in light of the previous formulae and of the remarks in $\S 5.2$ about toral elements of $U_{q}(\mathfrak{g l}(n+1))$, one has that

$$
\begin{gathered}
\varphi_{i} \text { is the } q \text {-analogue of } h_{i}, \\
\psi_{i} \text { is the } q \text {-analogue of } \ell_{i}, \\
\chi_{i} \text { is the } q \text {-analogue of } M_{i, i} ;
\end{gathered}
$$

on the other hand, the definition of these elements can also be motivated directly in quantum matrix terms. In the classical framework, we have

$$
h_{i}=M_{i, i}-M_{i+1, i+1}, \quad \ell_{i}=M_{1,1}+M_{2,2}+\cdots+M_{i, i}
$$

Now, $r_{i, i}$ is the $i$-th diagonal quantum matrix coefficient, that is the $q$-analogue of the "classical" matrix coefficient $M_{i, i}$; hence we should have, in principle,

$$
\left.\frac{r_{i, i}-1}{q-1}\right|_{q=1}=M_{i, i}
$$

such a relation is completely meaningful, and was our reason to define $\chi:=\frac{r_{i, i}-1}{q-1}$. As $G_{i}$ too is a $q$-analogue of $M_{i, i}$, the relation

$$
\xi_{P}\left(\chi_{i}\right)=\left(\begin{array}{c}
G_{i} ; 0 \\
1
\end{array}\right)+\mathcal{O}(q-1)
$$

is not surprising. By the way, we remark that the special relation

$$
\begin{aligned}
\sum_{i=1}^{n+1} r_{1,1} r_{2,2} \cdots & r_{i-1, i-1} \chi_{i}= \\
& =\sum_{\sigma \in S_{n+1} \backslash\{1\}}(-q)^{l(\sigma)}(q-1)^{e(\sigma)-1}\left(1+q^{-1}\right)^{e(\sigma)} r_{1, \sigma(1)} r_{2, \sigma(2)} \cdots r_{n+1, \sigma(n+1)}
\end{aligned}
$$

which arises from the relation $\operatorname{det}_{q}\left(\rho_{i j}\right)=1$, for $q=1$ turns into

$$
\chi_{1}+\chi_{2}+\cdots+\chi_{n+1}=0
$$

that is a relation like $\operatorname{Tr}(x)=0$.

Moreover, relations (5.2) should have quantum counterparts

$$
" q \text {-analogue of } h_{i} "=r_{i, i} \cdot r_{i+1, i+1}^{-1}, \quad " q \text {-analogue of } \ell_{i} "=r_{1,1} r_{2,2} \cdots r_{i, i},
$$

which should yield

$$
\left.\frac{r_{i, i} \cdot r_{i+1, i+1}^{-1}-1}{q-1}\right|_{q=1}=h_{i},\left.\quad \frac{r_{1,1} r_{2,2} \cdots r_{i, i}-1}{q-1}\right|_{q=1}=\ell_{i}
$$

now, the second relation in (5.3) is completely meaningful, so it moved us to define $\psi$ by $\psi_{i}:=\frac{r_{1,1} r_{2,2} \cdots r_{i, i}-1}{q-1}$; on the other hand, the first one instead is meaningless, for $r_{j, j}^{-1}$ does not exist; but since $r_{j, j} \equiv 1 \bmod (q-1)$, we should have also

$" q$-analogue of $h_{i} "=" q$-analogue of $h_{i} " \cdot r_{i+1, i+1}=r_{i, i} \cdot r_{i+1, i+1}^{-1} \cdot r_{i+1, i+1}=r_{i, i}$ 
whence the first relation in (5.4) turns into

$$
\left.\frac{r_{i, i}-r_{i+1, i+1}}{q-1}\right|_{q=1}=\left.\left(\frac{r_{i, i} \cdot r_{i+1, i+1}^{-1}-1}{q-1} \cdot r_{i+1, i+1}\right)\right|_{q=1}=h_{i}
$$

which provides a completely meaningful expression for a (tentative) $q$-analogue of $h_{i}$; that's why defined $\varphi_{i}:=\frac{r_{i, i}-r_{i+1, i+1}}{q-1}$. Notice that also $L_{i}$, resp. $K_{i}$, is a $q$-analogue of $\ell_{i}$, resp. $h_{i}$ : this explain the relation

$$
\xi_{P}\left(\psi_{i}\right)=\left(\begin{array}{c}
L_{i} ; 0 \\
1
\end{array}\right)+\mathcal{O}(q-1), \quad \operatorname{resp} . \quad \xi_{P}\left(\varphi_{i}\right)=\left(\begin{array}{c}
K_{i} ; 0 \\
1
\end{array}\right)+\mathcal{O}(q-1)
$$

Conclusion. The result of all the analysis above is that

$$
\begin{gathered}
\left.\xi_{P}\right|_{q=1}\left(\widetilde{F}_{q}^{P}[S L(n+1)] /(q-1) \widetilde{F}_{q}^{P}[S L(n+1)]\right)= \\
=\xi_{P}\left(\widetilde{F}_{q}^{P}[S L(n+1)]\right) /(q-1) \xi_{P}\left(\widetilde{F}_{q}^{P}[S L(n+1)]\right)=\mathfrak{U}^{P}(\mathfrak{h}) /(q-1) \mathfrak{U}^{P}(\mathfrak{h}), \\
\left.\xi_{Q}\right|_{q=1}\left(\widetilde{F}_{q}^{Q}[S L(n+1)] /(q-1) \widetilde{F}_{q}^{Q}[S L(n+1)]\right)= \\
=\xi_{Q}\left(\widetilde{F}_{q}^{Q}[S L(n+1)]\right) /(q-1) \xi_{Q}\left(\widetilde{F}_{q}^{Q}[S L(n+1)]\right)=\mathfrak{U}^{Q}(\mathfrak{h}) /(q-1) \mathfrak{U}^{Q}(\mathfrak{h}), \\
\left.\xi_{P}\right|_{q=1}\left(\widetilde{F}_{q}[S L(n+1)] /(q-1) \widetilde{F}_{q}[S L(n+1)]\right)= \\
=\xi_{P}\left(\widetilde{F}_{q}[S L(n+1)]\right) /(q-1) \xi_{P}\left(\widetilde{F}_{q}[S L(n+1)]\right)=\mathfrak{U}^{P}(\mathfrak{h}) /(q-1) \mathfrak{U}^{P}(\mathfrak{h}),
\end{gathered}
$$

because elements $F_{i},\left(\begin{array}{c}L_{i} ; 0 \\ 1\end{array}\right)$ - resp. $\left(\begin{array}{c}K_{i} ; 0 \\ 1\end{array}\right)$, resp. $\left(\begin{array}{c}G_{i} ; 0 \\ 1\end{array}\right)$ - and $E_{i}$ are enough to generate $\mathfrak{U}^{P}(\mathfrak{h}) /(q-1) \mathfrak{U}^{P}(\mathfrak{h})$, resp. $\mathfrak{U}^{Q}(\mathfrak{h}) /(q-1) \mathfrak{U}^{Q}(\mathfrak{h})$, resp. $\mathfrak{U}^{P}(\mathfrak{h}) /(q-1) \mathfrak{U}^{P}(\mathfrak{h})$. In particular, in this sense we claim that " $\widetilde{F}_{q}^{Q}[S L(n+1)]$, resp. $\widetilde{F}_{q}^{P}[S L(n+1)]$, is an approximation of $\mathcal{F}^{Q}[S L(n+1)]$, resp. $\mathcal{F}^{P}[S L(n+1)] "$.

It is worth stressing that this implies that Theorem 4.1 is a direct consequence of the specialization results about $\mathcal{F}^{P}[S L(n+1)]$ and $\mathcal{F}^{Q}[S L(n+1)]$ proved in [Ga], $\S 7$ (Theorem 7.3); conversely, those results follows from Theorem 4.1:

Theorem. $\mathcal{F}^{Q}[S L(n+1)]$ and $\mathcal{F}^{P}[S L(n+1)]$ specialize to $U(\mathfrak{h})$ as Poisson Hopf coalgebras for $q \rightarrow 1$. The same holds for $\mathfrak{U}^{Q}(\mathfrak{h})$ and $\mathfrak{U}^{P}(\mathfrak{h})$ too.

Proof. To be short let us set $\left.A\right|_{q=1}:=A /(q-1) A$ for any $k\left[q, q^{-1}\right]$-algebra. Now, we have $\widetilde{F}_{q}^{P}[S L(n+1)] \subseteq \mathcal{F}^{P}[S L(n+1)] \subseteq \mathfrak{U}^{P}(\mathfrak{h})$, and the analysis above shows - through and together with that in $[\mathrm{Ga}]$ - that $\left.\left.\widetilde{F}_{q}^{P}[S L(n+1)]\right|_{q=1} \cong \mathfrak{U}^{P}(\mathfrak{h})\right|_{q=1}$, hence

$$
\left.\left.\left.\widetilde{F}_{q}^{P}[S L(n+1)]\right|_{q=1} \cong \mathcal{F}^{P}[S L(n+1)]\right|_{q=1} \cong \mathfrak{U}^{P}(\mathfrak{h})\right|_{q=1}
$$

the same holds with $Q$ instead of $P$. Then the claim follows from Theorem 4.1. 


\section{$\S 6$ Generalization to $F_{q}[G L(n+1)]$}

6.1 The quantum matrix-function algebra $F_{q}[M(n+1)]$. We introduce the quantum matrix-function algebra of order $n+1(n \in \mathbb{N})$, to be called $F_{q}[M(n+1)]$, as follows.

By definition, $F_{q}[M(n+1)]$ is the associative $k(q)$-algebra with 1 generated by $\left\{x_{i j} \mid i, j=1, \ldots, n+1\right\}$ with relations

$$
\begin{aligned}
& x_{i j} x_{i k}=q x_{i k} x_{i j}, \quad x_{i k} x_{h k}=q x_{h k} x_{i k} \quad \forall j<k, i<h \\
& x_{i l} x_{j k}=x_{j k} x_{i l}, \quad x_{i k} x_{j l}-x_{j l} x_{i k}=\left(q-q^{-1}\right) x_{i l} x_{j k} \quad \forall i<j, k<l
\end{aligned}
$$

(that is, the same of $F_{q}^{P}[S L(n+1)]$ but for the relation "quantum determinant $=1$ "). This is a bialgebra, with co-operations defined by

$$
\Delta\left(x_{i j}\right)=\sum_{k=1}^{n} x_{i k} \otimes x_{k j}, \quad \epsilon\left(x_{i j}\right)=\delta_{i j} \quad \forall i, j .
$$

From the very definition we get a bialgebra epimorphism

$$
\pi: F_{q}[M(n+1)] \longrightarrow F_{q}^{P}[S L(n+1)], \quad x_{i j} \mapsto \rho_{i j} \quad \forall i, j
$$

6.2 The quantum function algebra $F_{q}[G L(n+1)]$. The element $\operatorname{det}_{q}\left(x_{i j}\right)$ of $F_{q}[M(n+1)]$ is group-like and central; thus by localization at $\operatorname{det}_{q}$ one can define a new algebra, namely $F_{q}[M(n+1)]\left[\right.$ det $\left._{q}^{-1}\right]$ : this is now a Hopf algebra, with bialgebra structure given by extension of that of $F_{q}[M(n+1)]$ and antipode defined by

$$
S\left(x_{i j}\right):=(-q)^{j-i} \operatorname{det}_{q}\left(\left(x_{h k}\right)_{h \neq j}^{k \neq i}\right) \quad \forall i, j .
$$

The Hopf algebra $F_{q}[G L(n+1)]:=F_{q}[M(n+1)]\left[\operatorname{det}_{q}^{-1}\right]$ is the quantum function algebra of the group $G L(n+1)$ (cf. [Ta]). It is clear that the bialgebra epimorphism $\pi: F_{q}[M(n+1)] \longrightarrow F_{q}^{P}[S L(n+1)]$ extends to $\pi: F_{q}[G L(n+1)] \longrightarrow F_{q}^{P}[S L(n+1)]$, a Hopf algebra epimorphism whose kernel is the (Hopf) ideal generated by $\left(\operatorname{det}_{q}\left(x_{i j}\right)-1\right)$.

The constructions and results in $\S \S 2-4$ can be easily extended to $F_{q}[G L(n+1)]$. It is straightforward to check that $k\left[q, q^{-1}\right]$-integer forms $\widetilde{F}_{q}^{P}[G L(n+1)]$ and $\widetilde{F}_{q}[G L(n+1)]$ of $F_{q}[G L(n+1)]$ can be defined mimicking the definitions of $\widetilde{F}_{q}^{P}[S L(n+1)]$ and $\widetilde{F}_{q}[S L(n+1)]$, with $x_{i j}$ 's instead of $\rho_{i j}$ 's: then one has a presentation of these algebras by generators and relations (namely, the same as for $\widetilde{F}_{q}^{P}[S L(n+1)]$, resp. $\widetilde{F}_{q}[S L(n+1)]$, but for the relation $\psi_{n+1}=-\sum_{\sigma \in S_{n+1} \backslash\{1\}}(-q)^{l(\sigma)}(q-1)^{e(\sigma)-1}\left(1+q^{-1}\right)^{e(\sigma)} r_{1, \sigma(1)} r_{2, \sigma(2)} \cdots r_{n+1, \sigma(n+1)}$, resp. $\quad \sum_{i=1}^{n+1} r_{1,1} r_{2,2} \cdots r_{i-1, i-1} \chi_{i}=\sum_{\sigma \in S_{n+1} \backslash\{1\}}(-q)^{l(\sigma)}(q-1)^{e(\sigma)-1}\left(1+q^{-1}\right)^{e(\sigma)}$. $\left.\cdot r_{1, \sigma(1)} r_{2, \sigma(2)} \cdots r_{n+1, \sigma(n+1)}\right)$. The upshot is that the specialization at $q=1$ of both of these integer forms is a Poisson Hopf coalgebra isomorphic to $U\left(\mathfrak{h}^{\prime}\right)$, where $\mathfrak{h}^{\prime}$ is the 
Lie bialgebra obtained by central extension of $\mathfrak{h}$ by an element $c$ (namely, $c=\left.\chi_{n+1}\right|_{q=1}$ ) such that

$$
\begin{gathered}
c x-x c=0 \quad \forall x \in \mathfrak{h} \quad \text { (i.e. } c \text { is central) } \\
\Delta(c)=c \otimes 1+1 \otimes c, \quad \epsilon(c)=0, \quad S(c)=-c \\
\delta(c)=4 \cdot \sum_{k=1}^{n} \mathrm{f}_{n+1, k} \wedge \mathrm{e}_{k, n+1}
\end{gathered}
$$

Thus again the quantum function algebra $F_{q}[G L(n+1)]$ can be seen as a quantum enveloping algebra, namely sort of a " $U_{q}\left(\mathfrak{h}^{\prime}\right)$ ".

\section{$\S 7$ PBW theorems}

In this section we shall prove some PBW theorems for $F_{q}^{P}[S L(n+1)]$ : that is, we shall exhibit some $k(q)$-basis of ordered monomials in the $\rho_{i j}$ 's for this algebra. To begin withs, we recall (cf. $[\mathrm{Ko}],[\mathrm{PW}]$ ) that, whenever we fix any total order in the set of generators $\left\{x_{i j} \mid i, j=1, \ldots, n+1\right\}$, the following PBW-type theorem holds for $F_{q}[M(n+1)]$ :

Proposition 7.1. The set of ordered monomials in the generators $x_{i j}$ 's is a $k(q)$-basis of $F_{q}[M(n+1)]$.

Now we wish to prove a similar result for $F_{q}^{P}[S L(n+1)]$; to this end we need a "triangularization argument", which is now explained. Define

$N_{+}:=k(q)$-subalgebra of $F_{q}[M(n+1)]$ generated by $\left\{x_{i j} \mid j<n+2-i\right\}$,

$N_{0}:=k(q)$-subalgebra of $F_{q}[M(n+1)]$ generated by $\left\{x_{i j} \mid j=n+2-i\right\}$,

$N_{-}:=k(q)$-subalgebra of $F_{q}[M(n+1)]$ generated by $\left\{x_{i j} \mid j>n+2-i\right\}$;

then we have the following result, whose proof easily follows from definitions and Proposition 7.1:

Proposition 7.2. Let any total order in $\left\{x_{i j} \mid i, j=1, \ldots, n+1\right\}$ be fixed. Then:

(a) the set of ordered monomials

$$
\left\{\prod_{j<n+2-i} x_{i j}^{m_{i j}} \mid m_{i j} \in \mathbb{N} \forall i, j\right\}
$$

is a $k(q)$-basis of $N_{+}$; the set of ordered monomials

$$
\left\{\prod_{i} x_{i, n+2-i}^{m_{i}} \mid m_{i} \in \mathbb{N} \forall i\right\}
$$

is a $k(q)$-basis of $N_{0}$; the set of ordered monomials

$$
\left\{\prod_{j>n+2-i} x_{i j}^{m_{i j}} \mid m_{i j} \in \mathbb{N} \forall i, j\right\}
$$

is a $k(q)$-basis of $N_{-}$;

(b) $N_{0}$ is a commutative subalgebra of $F_{q}[M(n+1)]$; 
(c) (Triangular Decomposition) the multiplication in $F_{q}[M(n+1)]$ gives a $k(q)$-vector space isomorphism

$$
F_{q}[M(n+1)] \cong N_{+} \otimes N_{0} \otimes N_{-}
$$

We are now ready for the first PBW theorem for $F_{q}^{P}[S L(n+1)]$.

Theorem 7.3 ( $1^{\text {st }}$ PBW theorem for $\left.F_{q}^{P}[S L(n+1)]\right)$. Let $\preceq$ be any fixed total ordering of the index set $\{(i, j) \mid i, j=1, \ldots, n\}$ such that $(i, j) \preceq(h, k) \preceq(l, m)$ for all $i, j, h$, $k, l, m$ such that $i<n+1-j, h=n+1-k, l>n+1-m$. Then the set of ordered monomials

$M^{\prime}:=\left\{\prod_{i<n+2-j} \rho_{i j}^{N_{i j}} \prod_{k=n+2-h} \rho_{h k}^{N_{h}} \prod_{l>n+2-m} \rho_{l m}^{N_{l m}} \mid N_{s t} \in \mathbb{N} \forall s, t ; \min \left\{N_{1}, \ldots, N_{n+1}\right\}=0\right\}$ is a $k(q)$-basis of $F_{q}^{P}[S L(n+1)]$.

Proof. We prove now that the above set does span $F_{q}^{P}[S L(n+1)]$; the linear independence will be an easy consequence of Theorem 7.4 below.

Since $F_{q}^{P}[S L(n+1)]$ is a homomorphic image of $F_{q}[M(n+1)]$, it is clear that the whole set (without restriction on the indices $N_{h}$ 's) of ordered monomials in the $\rho_{i j}$ 's does span $F_{q}^{P}[S L(n+1)]$ over $k(q)$. Now pick up any monomial

$$
m(\underline{N}):=\prod_{i<n+2-j} \rho_{i j}^{N_{i j}} \cdot \prod_{k=n+2-h} \rho_{h k}^{N_{h}} \cdot \prod_{l>n+2-m} \rho_{l m}^{N_{l m}}
$$

such that $d:=\min \left\{N_{1}, \ldots, N_{n+1}\right\}>0$; since the generators $\rho_{h, n+2-h}(h=1 \ldots, n+1)$ commute with each other, we can single out of the " $\pi\left(N_{0}\right)$-part" (with respect to the triangular decomposition inherited from that in Proposition 7.2(c)) $n_{0}:=\prod_{k=n+2-h} \rho_{h k}^{N_{h}}=$ $\prod_{h=1}^{n+1} \rho_{h, n+2-h}^{N_{h}}$ of $m(\underline{N})$ a factor $\rho_{1, n+1} \rho_{2, n} \cdots \rho_{n+1,1}$, and we can do it $d$ times. Now using the relation $\operatorname{det}_{q}\left(\rho_{i j}\right)-1=0$ we substitute the factor $\rho_{1, n+1} \rho_{2, n} \cdots \rho_{n+1,1}$ in $m(\underline{N})$ with

$$
(-q)^{\left(\begin{array}{c}
n+1 \\
2
\end{array}\right)}-\sum_{\sigma \in S_{n+1} \backslash\left\{w_{0}\right\}}(-q)^{\left(\begin{array}{c}
n+1 \\
2
\end{array}\right)-\ell(\sigma)} \cdot \rho_{1, \sigma(1)} \rho_{2, \sigma(2)} \cdots \rho_{n+1, \sigma(n+1)}
$$

where $w_{0}$ is the longest element of $S_{n+1}$; now look at the various summands $\rho_{1, \sigma(1)} \rho_{2, \sigma(2)} \cdots \rho_{n+1, \sigma(n+1)}$ (up to the proper coefficient) coming in, with $\sigma \neq w_{0}$ : whenever we have $\sigma(j+1)<\sigma(j)$ the commutation rules give

$$
\rho_{j, \sigma(j)} \rho_{j+1, \sigma(j+1)}=\rho_{j+1, \sigma(j+1)} \rho_{j, \sigma(j)}
$$

in particular, if $j \geq n+2-\sigma(j)$ and $j+1 \leq n+2-\sigma(j+1)$ we have exactly $\rho_{j, \sigma(j)} \rho_{j+1, \sigma(j+1)}=\rho_{j+1, \sigma(j+1)} \rho_{j, \sigma(j)}$; it follows that we can factor out the monomial $\rho_{1, \sigma(1)} \rho_{2, \sigma(2)} \cdots \rho_{n+1, \sigma(n+1)}$ as

$$
\rho_{1, \sigma(1)} \rho_{2, \sigma(2)} \cdots \rho_{n+1, \sigma(n+1)}=n_{+} \cdot n_{0}^{\prime} \cdot n_{-}
$$


with $n_{+} \in \pi\left(N_{+}\right), n_{0}^{\prime} \in \pi\left(N_{0}\right), n_{-} \in \pi\left(N_{-}\right)$, and $\operatorname{deg}\left(n_{0}^{\prime}\right)<\operatorname{deg}\left(n_{0}\right)$ as monomials in the $\rho_{i j}$ 's. Therefore at the end we are left with a new expression of $m(\underline{N})$ as a linear combination of monomials which, with respect to the triangular decomposition inherited from Proposition $7.3(\mathrm{c})$, have a " $\pi\left(N_{0}\right)$-part" of lower degree; then a simple induction argument finishes the proof. 
We conclude with our second PBW theorem: this is the most interesting, because it is directly related to the classical PBW theorem attached to the natural triangular decomposition of $U(\mathfrak{h})$.

Theorem 7.4 ( $2^{\text {nd }}$ PBW theorem for $\left.F_{q}^{P}[S L(n+1)]\right)$. Let $\preceq$ be any fixed total ordering of the index set $\{(i, j) \mid i, j=1, \ldots, n\}$ such that $(i, j) \preceq(h, k) \preceq(l, m)$ for all $i, j, h, k$, $l, m$ such that $i>j, h=k, l<m$. Then the set of ordered monomials

$$
M:=\left\{\prod_{i>j} \rho_{i j}^{N_{i j}} \prod_{h=k} \rho_{h k}^{N_{h k}} \prod_{l<m} \rho_{l m}^{N_{l m}} \mid N_{s t} \in \mathbb{N} \forall s, t ; \min \left\{N_{1,1}, \ldots, N_{n+1, n+1}\right\}=0\right\}
$$

is a $k(q)$-basis of $F_{q}^{P}[S L(n+1)]$.

Proof. As $F_{q}[M(n+1)]$ is clearly $\mathbb{N}^{(n+1)^{2}}$-graded (by the degree in each variable), it is also $\mathbb{N}$-graded (by the total degree); hence $F_{q}^{P}[S L(n+1)]$ inherits a filtration, arising from the filtration of $F_{q}[M(n+1)]$ associated to the $\mathbb{N}$-grading, say

$$
F_{0} \subset F_{1} \subset F_{2} \subset \cdots \subset F_{r} \subset \cdots \subset F_{q}^{P}[S L(n+1)]=\bigcup_{r=0}^{+\infty} F_{r} ;
$$

furthermore, we have $M=\cup_{r=0}^{+\infty} M_{r}$, with $M_{r}:=M \cap F_{r}$ for all $r \in \mathbb{N}$.

Similarly we have $M^{\prime}=\cup_{r=0}^{+\infty} M_{r}^{\prime}$, with $M_{r}^{\prime}:=M^{\prime} \cap F_{r}$ for all $r \in \mathbb{N}$, where $M^{\prime}$ is the set of ordered monomials defined in Proposition 7.4 above: in particular, $M_{r}^{\prime}$ spans $F_{r}$ over $k(q)$. Finally the very definitions ensure that

$$
\#\left(M_{r}\right)=\#\left(M_{r}^{\prime}\right) \quad \forall r \in \mathbb{N} .
$$

Now consider the specialization $F_{q}^{P}[S L(n+1)] \stackrel{q \rightarrow 1}{\longrightarrow} F[S L(n+1)]$ and the corresponding set $M^{(1)}$ of "specialized monomials", i.e. the image of $M$ under the epimorphism $F_{q}^{P}[S L(n+1)] \longrightarrow F_{1}^{P}[S L(n+1)]=F[S L(n+1)]:$ if we prove that $M^{(1)}$ is a linearly independent set (over $k$ ), then the same will be true for $M$ (over $k(q)$ ); in particular $M_{r}$ will be linearly independent $(\forall r \in \mathbb{N})$, hence it will be a $k(q)$-basis of $F_{r}(\forall r \in \mathbb{N})$, because of (7.2), whence finally $M$ will be a $k(q)$-basis of $F_{q}^{P}[S L(n+1)]$. Thus let us prove that the set $M^{(1)}$ is linearly independent over $k$.

Assume we have in $F_{1}^{P}[S L(n+1)]=F[S L(n+1)]$ a relation

$$
\sum_{m \in M^{(1)}} a_{m} \cdot m=0
$$

for some (finitely many) $a_{m} \in k \backslash\{0\}$; then (7.3) lifts up to a relation in $k\left[\left\{z_{i j}\right\}_{i, j=1, \ldots, n+1}\right]$

$$
\sum_{m \in M^{(1)}} a_{m} \cdot m(z)=b(z) \cdot\left(\operatorname{det}\left(z_{i j}\right)-1\right)
$$

for some $b(z) \in k\left[\left\{z_{i j}\right\}_{i, j=1, \ldots, n+1}\right]$, the $m(z)$ 's having the obvious meaning. If $b(z)=0$, then (7.4) gives a non-trivial algebraic relation among the $z_{i j}$ 's, which is impossible; if $b(z) \neq 0$, then the right-hand-side of (7.4) involves - with non-zero coefficient - the monomial $z_{1,1} z_{2,2} \cdots z_{n+1, n+1}$ (coming out of $\operatorname{det}\left(z_{i j}\right)$ ), while each monomial $m(z)$ in the left-hand-side does not contain the factor $z_{1,1} z_{2,2} \cdots z_{n+1, n+1}$; thus again (7.4) yields a non-trivial algebraic relation among the $z_{i j}$ 's, which is impossible. The claim follows. 


\title{
REFERENCES
}

[APW] H. H. Andersen, P. Polo, Wen Kexin, Representations of quantum algebras, Invent. Math. 104 (1991), 1-59.

[DKP] C. De Concini, V. G. Kac, C. Procesi, Quantum coadjoint action, Jour. Am. Math. Soc. 5 (1992), 151 - 189.

[DL] C. De Concini, V. Lyubashenko, Quantum function algebra at roots of 1, Adv. Math. 108 (1994), $205-262$.

[DP] C. De Concini, C. Procesi, Quantum groups, in L. Boutet de Monvel, C. De Concini, C. Procesi, P. Schapira, M. Vergne (eds.), D-modules, Representation Theory, and Quantum Groups, Lectures Notes in Mathematics 1565, Springer \& Verlag, Berlin-Heidelberg-New York, 1993.

[Dr] V. G. Drinfeld, Quantum groups, Proc. ICM Berkeley 1 (1986), 789-820.

[Ga] F. Gavarini, Quantization of Poisson groups, to appear in Pac. Jour. Math. (preprint q-alg/9604007).

[GL] I. Grojnowski, G. Lusztig, On bases of irreducible representation of quantum group $G L_{n}$, in V. Deodhar (ed.), Kazhdan-Lusztig theory and related topics, Cont. Math. 139 (1992), 167-174.

[Ko] H. T. Koelink, On $*$-representations of the Hopf $*$-algebra associated with the quantum group $U_{q}(n)$, Compositio Mathematicae $7 \mathbf{7}$ (1992), 199-231.

[Ji] M. Jimbo, A q-analogue of $U(g l(N+1))$, Hecke Algebras and the Yang Baxter equation, Lett. Math. Phys. 10 (1985), 63-69.

[Pa] P. Papi, A characterization of a good ordering in a root system, Proc. Amer. Math. Soc. 120 (1994), 661-665.

[PW] B. Parshall, J. Wang, Quantum linear groups, Mem. Amer. Math. Soc. 89 (1991), $n^{O} 439$.

[Ta] M. Takeuchi, Some topics on $G L_{q}(n)$, J. Algebra 147 (1992), 379-410.

\author{
Institut De ReCherche Mathématique Avancée \\ Université "Louis PASTEUR" - C.N.R.S. \\ 7, Rue René Descartes \\ 67084 STRASBOURG CEDEX — FRANCE \\ E-MAIL: GAVARINI@MATH.U-STRASBG.FR \\ GAVARINI@MAT.UNIROMA1.IT \\ GAVARINI@MAT.UNIROMA3.IT
}

